\title{
Flexible contracts*
}

\author{
Piero Gottardi \\ European University Institute \\ Jean Marc Tallon \\ Paris School of Economics, CNRS \\ Paolo Ghirardato \\ Università di Torino
}

This version: December 3, 2015

\begin{abstract}
This paper studies the costs and benefits of delegating decisions to superiorly informed agents, that is of adopting flexible contracts, relative to the use of rigid, non discretionary contracts. The main focus of the paper lies in the analysis of the costs of delegation, primarily agency costs, versus their benefits, primarily the flexibility of the action choice in two different environments, one with risk and one with ambiguity.

We first determine and characterize the properties of the optimal flexible contract. We then show that the higher the agent's degree of risk aversion, the higher is the agency costs of delegation and the less profitable a flexible contract relative to a rigid one. When the parties have imprecise probabilistic beliefs, the agent's degree of imprecision aversion introduces another agency cost, which again reduces the relative profitability of flexible contracts.
\end{abstract}

JEL Classification: D86, D82, D81.

Keywords: Delegation, Flexibility, Agency Costs, Multiple Priors, Imprecision Aversion.

\footnotetext{
${ }^{*}$ We wish to thank B. Lipman, B. Salanié and especially D. Martimort for helpful discussions. We are also grateful to an Associate Editor and two anonymous referees for useful comments. Gottardi: Department of Economics, European University Institute, Villa San Paolo, Via della Piazzuola 43, 50133 Firenze. Email: piero.gottardi at EUI.eu. Tallon: Centre d'Economie de la Sorbonne, 106 bld de l'Hôpital, 75013 Paris. Email jmtallon at univ-paris1.fr. Ghirardato: Dipartimento ESOMAS and Collegio Carlo Alberto, Via Real Collegio 30, 10024 Moncalieri. Email: paolo.ghirardato at carloalberto.org. Gottardi and Tallon acknowledge support from, respectively, the EUI Research Council and the French National Research Agency AmGames, projet ANR 12-FRAL-0008-01 as well as ANR-10-LABX-93-01. Ghirardato acknowledges support from the PRIN 2010-2011 grant "Robust Decision Making in Markets and Organizations" by the Italian MIUR.
} 


\section{Introduction}

Motivation. A central problem in organizations is the fact that, as argued by Jensen and Meckling (1992), agents assigned a given task may end up having, at the time they have to act, some superior information on the suitability of the various actions which can be taken to perform the assigned task. As a consequence, it may be desirable, in order to enhance the performance of the organization, to grant agents some degree of discretion in their choice of which action to undertake, or to ask them to report their information before specifying which action should be carried out. The obvious difficulty in doing this is that the interests of such agents may not be aligned with those of the organization. This difficulty can be mitigated and possibly eliminated with the use of appropriate monetary transfers to the agents, that is of appropriate compensation contracts. For such contracts to work, some risk must be typically shifted to the agents. If agents are risk averse, doing this is costly. Moreover, if the nature of the possible realizations of the uncertainty is not clearly understood a priori, either because some unforeseen contingencies may arise or because the probabilities of the possible events may be 'ambiguous,' some further difficulties and costs arise.

The presence of these costs implies that, in the decision of whether or not and to which extent to delegate to an agent the choice of which action to undertake, a trade-off is faced. The broader the uncertainty about the environment in which the agent takes his action, the more important is for the organization the fact that the 'right' action is taken in each possible circumstance, and the higher are the benefits of delegating the choice to the agent, that is of offering him a contract granting some flexibility in his choice. But the extent and nature of this uncertainty also affect the costs of delegation. These costs depend on the risk aversion of the agent, as well as on the degree of 'ambiguity' of such uncertainty and the attitude towards it exhibited by the agent. When such costs are sufficiently high it might be preferable to opt for a different type of contract, which does not delegate the action choice to the agent.

The issue is important, as this trade-off naturally arises when the architecture of organizations is evaluated. The main focus of this paper is the analysis of this tradeoff, in particular of how the cost of delegating decisions to superiorly informed agents varies with the structure of the uncertainty and the agents' attitude towards risk and uncertainty. 
Model and results. We consider a simple contracting situation between a principal and an agent. The agent must take a costly action which generates some revenue for the principal. Before taking his action, but after signing the contract, the agent receives a private signal over the productivity of the various actions. The action chosen by the agent is not observable by the principal but we assume that, at the time of contracting, the principal has the ability to predefine the set of actions, or possible tasks, available to the agent. Thus the principal could specify a determinate action the agent must undertake in all the possible circumstances he may have to act - what we will call a rigid, or non discretionary, contract. ${ }^{1}$ Alternatively, the principal could leave the agent some discretion in his choice, so that the action the agent undertakes may vary with the information received - a flexible contract.

The agent incurs a (deterministic) cost in order to undertake each of the possible actions. Hence, in the absence of monetary transfers contingent on the realization of the principal's revenue the interests of the principal and the agent may not be aligned as the latter would always choose the least costly action among those available to him. A flexible contract must therefore include a suitably designed compensation scheme, in order to induce the agent to take the revenue maximizing action for each realization of the signal. But such variability in compensation may generate agency costs. In contrast, a rigid contract is simpler, does not need to rely on high-powered incentives and never entails agency costs.

Given the important role played by the uncertainty faced by the parties concerning future events, we will consider and compare two different specifications of the parties' information and attitude towards this uncertainty. Consider first the case where principal and agent have common and sharp probabilistic beliefs about the possible circumstances in which the agent will have to act and about the productivity of the different actions. In this environment, if the agent is risk neutral, ${ }^{2}$ agency costs are zero and the optimal flexible contract always dominates, at least weakly, the rigid contract. This is no longer true if the agent is risk averse, as then agency costs arise. We characterize the optimal flexible contract when the agent has CARA preferences, so as to be able to isolate the effects of changes in the agent's degree of risk aversion. We find that at the optimal

\footnotetext{
${ }^{1}$ The possibility of imposing such restrictions was earlier considered in various papers starting with Holmstrom (1984) (see Alonso and Matouschek (2008), Armstrong and Vickers (2009) for some recent contributions) in which actions are assumed observable and no monetary transfers are allowed.

${ }^{2} \mathrm{We}$ assume the principal is always risk neutral.
} 
flexible contract the agent's compensation also depends on the agent's report over the signal received and that the agent's utility is not equalized across different realizations of the signal. Also, an increase in the agent's degree of (absolute) risk aversion implies a larger agency cost, and hence a lower profitability for the principal of a flexible contract relative to a rigid contract. Thus, there is a threshold level for the agent's degree of risk aversion, above which a rigid contract dominates a flexible one and below which the opposite is true.

We next turn our attention to environments in which the information available to the parties concerning the possible events in which the agent is called to act is not precise enough to pin down a single probability distribution; i.e. there is ambiguity. This might for instance be the case if the circumstances under which the agent may have to choose an action are totally new, with almost no information available. Or it might be due to the fact that these circumstances are hard to describe in full detail. We model this fact by assuming that principal and agent have a common -i.e., objective- set of probabilistic beliefs over the likelihood of these events. Following Gajdos, Hayashi, Tallon, and Vergnaud (2008), we call 'imprecision' such set of beliefs and 'imprecision averse' a decision maker who reacts pessimistically to such imprecision, and 'imprecision neutral' a decision maker who anyway condenses the imprecise set to a single probability. ${ }^{3}$ Specifically, we assume that the principal is imprecision neutral while the agent is imprecision averse. To single out the effect of the presence of imprecision, we assume here that both parties are risk neutral and show that imprecision aversion by itself creates an agency cost. We provide a partial characterization of the optimal flexible contract under imprecision aversion and show that increasing the agent's degree of imprecision aversion reduces the profits of the optimal flexible contract, making the rigid contract relatively more attractive. We also find that the properties of the optimal flexible contract under imprecision aversion, in particular with regard to the variability of the compensation paid to the agent across different realizations of the uncertainty, are different from those obtained under risk aversion.

Even though with multiple priors the compensation contract may be designed so that principal and agent end up 'using different beliefs' to evaluate it, and hence possibly engage in mutually beneficial speculative trade, we show this is never optimal. This stands in contrast with the case in which both principal and agent have sharp, but different prior

\footnotetext{
${ }^{3}$ We remark that with some differences in interpretation, our analysis could be equivalently cast in the original maxmin expected utility model of Gilboa and Schmeidler (1989), or its $\alpha$-maxmin generalization (e.g., Ghirardato, Maccheroni, and Marinacci (2004)).
} 
beliefs, where the surplus generated by the contractual relationship is actually enhanced by the possibility of exploiting the benefits of speculative trade (as in Eliaz and Spiegler (2007)).

\section{Literature.}

The choice between flexible and rigid contractual structures in organizations has been examined in several previous papers. In contrast to our setup, most of these papers focus on the case where monetary transfers are not allowed and the objectives of principal and agent are at least partly aligned. In such environments the agent may be willing to freely transmit some of his private information to the principal. Dessein (2002) investigates the trade-off between contracts where the choice of the action is delegated to the agent and contracts where the principal retains the control over such choice, but uses the information that is reported to him by the agent. He examines in particular how such trade-off varies with the degree of congruence between the objectives of the principal and the agent. Aghion and Tirole (1997) and Szalay (2005) study the consequences, on the agent's incentives to invest in acquiring information, of delegating -possibly only partial- control over the action choice to the agent .

The closest paper to ours is Prendergast (2002). Like us, he considers an environment in which monetary transfers are allowed, the structure of information is given and the agent has superior information. In his set-up the agent is risk neutral like the principal, and agency costs are exogenously given (as fixed 'monitoring costs'). He examines how the relative benefits of flexible and rigid contracts vary with respect to the magnitude of the uncertainty facing the agent, that is to the variability in the possible situations in which he may find himself to act. In contrast, our main focus is the endogenous determination of the agency costs, how they vary with the agent's attitude to uncertainty, and their effects for the properties of the optimal contract.

Following up on Prendergast's work, Baker and Jorgensen (2003) study the effects on the steepness of the agent's incentives of the precision of the signal privately observed by the agent on the productivity of his effort. The analysis is carried out in a CARA normal environment, restricting attention to linear compensation contracts which only depend on the output level. They find that the steepness of the incentives increases with the volatility of the signal. We consider a simpler environment, in which it is possible to characterize the optimal contract without imposing restrictions on its functional form; we also allow the contract to depend on the agent's report over his information. Moreover, 
the main focus of our analysis is on the trade-off between flexible and rigid contracts rather than the steepness of incentives. Raith (2008) considers an environment where the agent has superior information over the productivity of its effort, while his effort is observable. The main goal of his analysis is the characterization of the optimal contract, again restricting attention to linear compensation contracts in both effort and output. ${ }^{4}$ In contrast, in our set-up the agent's action is only observable when it is predetermined, that is, with no delegation.

A rather different characterization of the trade-off between rigidity and flexibility is provided by Hart and Moore (2008), where the main cost of delegation lies in the variability of the outcome prescribed by the contract and the deadweight losses this generates.

The effects of ambiguity or imprecision in the probabilistic beliefs concerning the possible realizations of the environment faced by parties in contractual situations have been first examined by Mukerji (1998) and Ghirardato (1994). Mukerji (1998) studies a vertical relationship problem, using the Choquet expected utility model of Schmeidler (1989). He shows that, as a result of ambiguity aversion, the optimal contract might be incomplete and, differently from our setup, exhibit low powered incentives. Ghirardato (1994) looks at a standard moral hazard problem where parties' 'beliefs' are non-additive and reflect ambiguity aversion: each action taken by the agent induces a non-additive distribution on outcomes. While he also discusses the nature of agency costs in such environment, he does not address the question of delegation.

The paper is organized as follows. The next section describes the environment. Section 3 presents the contracting problem under risk, characterizing its solution and outlining the trade-off between flexible and rigid contracts. Section 4 extends the analysis to the case where the parties do not have precise probabilistic beliefs. Section 5 offers some concluding remarks. Proofs are collected in the Appendix.

\section{The set-up}

We consider a contractual relationship between a principal, say a firm, and an agent, say a worker. The worker has two possible actions, $x$ and $y$. The output generated by each action is uncertain: it can be either high $(\bar{R})$ or low $(\underline{R})$. The probability of the different

\footnotetext{
${ }^{4}$ See also Rantakari (2008).
} 
output realizations when a certain action is undertaken is also uncertain and depends on some event $\theta \in\left\{\theta_{1}, \theta_{2}\right\}$.

The realization of the output is publicly observable while the action chosen by the agent is only privately known to him. Furthermore the realization of $\theta$, describing an event affecting the probability of success (high output) of the different possible actions, is privately observed by the agent before his action is chosen. It is not observed by the principal nor by any third party. To begin with, we examine the case where both principal and agent have sufficient information over the process generating this uncertainty, and are thus able to come up with a sharp probabilistic belief over it. Let $p$ denote their common belief concerning the occurrence of $\theta_{1}$ and $\pi(x, \theta)$ (resp. $\pi(y, \theta)$ ) denote the probability that $R=\bar{R}$ in event $\theta$ when action $x$ (resp. $y$ ) is chosen.

The contract is written before the realization of any source of uncertainty (i.e., before the output and $\theta$ are realized). Although the action undertaken by the agent is not observable, we assume that, at the time the contract is signed, the principal can impose some restrictions over the set of actions available to the agent. To understand the nature of these restrictions we can think, for instance, of a situation where the principal can decide to install either only one software program on the agent's computer (in which case only one action is available to the agent) or different types of software. In the latter case the agent is free to choose which software to use ( $x$ or $y)$ to perform the task and his actual choice is not observable. Also, the fact that such restrictions can only be imposed ex ante can be justified if we think of situations where the timing of the resolution of the uncertainty over $\theta$, and hence of the action choice, is also uncertain and privately observed.

In this framework, a contract is a specification of a set of admissible actions $A \subseteq\{x, y\}$ together with a wage payment $w$ from the principal to the agent, where $w$ can depend on the realized level of the output and the agent's announcement about the realization of the event $\theta$. Let $\bar{w}_{i}$ (resp. $\underline{w}_{i}$ ) denote the compensation paid to the agent when the output is $\bar{R}$ (resp. $\underline{R}$ ) and the (declared) state is $\theta_{i}, i=1,2$.

In particular, we would like to distinguish the case where the full menu of possible actions is available to the agent, $A=\{x, y\}$, from the cases where only action $x$ - or only action $y$ - is available to the agent. We refer to the contract in the first case as a flexible contract, since the agent has the flexibility and the discretion to choose the action he thinks is more appropriate for him (and suitable incentives should be specified in the 
contract to induce the agent to make a choice also in the principal's interest). In the second case we say on the other hand the contract is rigid, as it prescribes the agent to always undertake a given action. The contract can then be of type $x$ or of type $y$ according to which action is specified.

The time-line is as follows:

$t=0$ The contract is signed, specifying the set $A$ of possible actions available to the agent and his compensation $w$.

$t=1 \theta$ is observed by the agent who announces its value to the principal.

$t=2$ The agent undertakes an action.

$t=3$ Output is revealed

$t=4$ Compensation is paid to the agent, according to the realized output level and the agent's announcement.

Observe that at the time the contract is signed there is symmetric information among the parties. Asymmetric information will arise at a later stage, when the agent privately learns the realization of $\theta$, affecting the profitability of the different actions, and chooses then which action to take.

Remark 1 We ignore here the possibility of renegotiation, in particular at $t=1$, after the agent learns the realization of $\theta$.

The principal is the residual claimant of the output and is risk neutral. His payoff, when action $z_{i} \in A$ is implemented in state $\theta_{i}, i=1,2$, is given by the expected profit:

$$
\begin{aligned}
p\left[\pi\left(z_{1}, \theta_{1}\right)\left(\bar{R}-\bar{w}_{1}\right)+\right. & \left.\left(1-\pi\left(z_{1}, \theta_{1}\right)\right)\left(\underline{R}-\underline{w}_{1}\right)\right] \\
& +(1-p)\left[\pi\left(z_{2}, \theta_{2}\right)\left(\overline{\bar{R}}-\bar{w}_{2}\right)+\left(1-\pi\left(z_{2}, \theta_{2}\right)\right)\left(\underline{R}-\underline{w}_{2}\right)\right]
\end{aligned}
$$

The agent has a non separable ${ }^{5}$ utility function over the compensation received and the $\operatorname{cost} c_{z}$ of undertaking the action $z \in A$ that is chosen. In particular, in most of the paper we will assume the agent is risk averse and exhibits the following preferences:

\footnotetext{
${ }^{5} \mathrm{~A}$ utility function that is non separable in the wage received and the cost incurred allows us to study the comparative statics properties of the optimal contract with respect to the agent's degree of risk aversion - one of our objectives. In fact with such a specification the rate of substitution between actions and wage payments is constant and changes in the curvature of the agent's utility function only capture changes in the agent's attitude towards risk in his compensation.
} 
Assumption 1 The agent has a CARA utility function: $u(w, z)=-\frac{e^{-a\left(w-c_{z}\right)}}{a}$, with $a>0$.

The agent's risk attitude is described by the parameter $a$. It is then convenient to state the agent's reservation utility as $-\frac{e^{-a \bar{u}}}{a}$.

We will also assume:

\section{Assumption 2}

i) $\Delta c \equiv c_{x}-c_{y}>0$,

ii) $\pi\left(x, \theta_{1}\right)>\pi\left(x, \theta_{2}\right)>\pi\left(y, \theta_{2}\right)>\pi\left(y, \theta_{1}\right)$,

iii) $\left(\pi\left(x, \theta_{1}\right)-\pi\left(y, \theta_{1}\right)\right)(\bar{R}-\underline{R})>\Delta c>\left(\pi\left(x, \theta_{2}\right)-\pi\left(y, \theta_{2}\right)\right)(\bar{R}-\underline{R})$,

iv) $\frac{1-\pi\left(y, \theta_{1}\right)}{1-\pi\left(x, \theta_{1}\right)} \geq e^{a \Delta c}$.

Conditions i) and ii) say that action $x$ is both more costly and more productive than action $y$. Also, condition ii) together with condition iii) say that the additional productivity of action $x$, relative to action $y$, is uncertain: it is larger in state $\theta_{1}$ than in state $\theta_{2}$ and this variability in the productivity differential is sufficiently significant that in state $\theta_{1}$ the expected revenue net of the cost is higher for action $x$ and in state $\theta_{2}$ it is higher for action $y$. Hence conditions i-iii) ensure that, if there were no agency problems (that is, if both $\theta$ and the agent's action were publicly observed), the optimal contract would be a flexible one, implementing action $x$ in $\theta_{1}$ and acion $y$ in $\theta_{2}$.

Finally, condition iv) says that in state $\theta_{1}$ the productivity differential of action $x$ relative to $y$ is sufficiently large, relative to the utility cost of effort. It ensures, as we will see, that the agency costs are not too high and hence that the profile of actions $x$ in $\theta_{1}$ and $y$ in $\theta_{2}$ is implementable even when the state $\theta$ and the agent's actions are only privately observed.

In addition, we should point out that condition ii) says that at least one of the two actions is more productive in state $\theta_{2}$ than in state $\theta_{1}$. As explained in Remark 3 below, this property ensures that the agent's private information over the realization of state $\theta$ matters, while with alternative specifications of the probabilities, still consistent with the other conditions in Assumption 2 but violating this property, the optimal contract is the same as when $\theta$ is publicly observable. 


\section{Optimal Contract under risk}

\subsection{Optimal flexible contract}

The advantage of a flexible contract over a rigid one is that it allows to implement the action profile that maximizes net revenue, which under Assumption 2 is given by action $x$ in $\theta_{1}$ and $y$ in $\theta_{2}$. The cost is that, to implement such an action profile, appropriate incentive constraints need to be imposed, ensuring that no possible deviation by the agent, in his action choice and/or reporting over the state, is profitable. The optimal contract implementing this action profile subject to the incentive constraints (to which we will refer, with a slight abuse of terminology, as the optimal flexible contract) is obtained as solution of the following programme:

$$
\begin{aligned}
& \max _{\bar{w}_{1}, \underline{w}_{1}, \bar{w}_{2}, \underline{w}_{2}} \quad p\left[\pi\left(x, \theta_{1}\right)\left(\bar{R}-\bar{w}_{1}\right)+\left(1-\pi\left(x, \theta_{1}\right)\right)\left(\underline{R}-\underline{w}_{1}\right)\right] \\
& +(1-p)\left[\pi\left(y, \theta_{2}\right)\left(\bar{R}-\bar{w}_{2}\right)+\left(1-\pi\left(y, \theta_{2}\right)\right)\left(\underline{R}-\underline{w}_{2}\right)\right] \\
& \text { s.t. } \\
& \left\{\begin{array}{c}
(I C 1)-\pi\left(x, \theta_{1}\right) e^{-a\left(\bar{w}_{1}-c_{x}\right)}-\left(1-\pi\left(x, \theta_{1}\right)\right) e^{-a\left(\underline{w}_{1}-c_{x}\right)} \geq-\pi\left(x, \theta_{1}\right) e^{-a\left(\bar{w}_{2}-c_{x}\right)}-\left(1-\pi\left(x, \theta_{1}\right)\right) e^{-a\left(\underline{w}_{2}-c_{x}\right)} \\
(I C 2)-\pi\left(x, \theta_{1}\right) e^{-a\left(\bar{w}_{1}-c_{x}\right)}-\left(1-\pi\left(x, \theta_{1}\right)\right) e^{-a\left(\underline{w}_{1}-c_{x}\right)} \geq-\pi\left(y, \theta_{1}\right) e^{-a\left(\bar{w}_{1}-c_{y}\right)}-\left(1-\pi\left(y, \theta_{1}\right)\right) e^{-a\left(\underline{w}_{1}-c_{y}\right)} \\
(I C 3)-\pi\left(x, \theta_{1}\right) e^{-a\left(\bar{w}_{1}-c_{x}\right)}-\left(1-\pi\left(x, \theta_{1}\right)\right) e^{-a\left(\underline{w}_{1}-c_{x}\right)} \geq-\pi\left(y, \theta_{1}\right) e^{-a\left(\bar{w}_{2}-c_{y}\right)}-\left(1-\pi\left(y, \theta_{1}\right)\right) e^{-a\left(\underline{w}_{2}-c_{y}\right)} \\
(I C 4)-\pi\left(y, \theta_{2}\right) e^{-a\left(\bar{w}_{2}-c_{y}\right)}-\left(1-\pi\left(y, \theta_{2}\right)\right) e^{-a\left(\underline{w}_{2}-c_{y}\right)} \geq-\pi\left(y, \theta_{2}\right) e^{-a\left(\bar{w}_{1}-c_{y}\right)}-\left(1-\pi\left(y, \theta_{2}\right)\right) e^{-a\left(\underline{w}_{1}-c_{y}\right)} \\
(I C 5)-\pi\left(y, \theta_{2}\right) e^{-a\left(\bar{w}_{2}-c_{y}\right)}-\left(1-\pi\left(y, \theta_{2}\right)\right) e^{-a\left(\underline{w}_{2}-c_{y}\right)} \geq-\pi\left(x, \theta_{2}\right) e^{-a\left(\bar{w}_{2}-c_{x}\right)}-\left(1-\pi\left(x, \theta_{2}\right)\right) e^{-a\left(\underline{w}_{2}-c_{x}\right)} \\
(I C 6)-\pi\left(y, \theta_{2}\right) e^{-a\left(\bar{w}_{2}-c_{y}\right)}-\left(1-\pi\left(y, \theta_{2}\right)\right) e^{-a\left(\underline{w}_{2}-c_{y}\right)} \geq-\pi\left(x, \theta_{2}\right) e^{-a\left(\bar{w}_{1}-c_{x}\right)}-\left(1-\pi\left(x, \theta_{2}\right)\right) e^{-a\left(\underline{w}_{1}-c_{x}\right)} \\
(P C)-p\left[\pi\left(x, \theta_{1}\right) e^{-a\left(\bar{w}_{1}-c_{x}\right)}+\left(1-\pi\left(x, \theta_{1}\right)\right) e^{-a\left(\underline{w}_{1}-c_{x}\right)}\right]- \\
(1-p)\left[\pi\left(y, \theta_{2}\right) e^{-a\left(\bar{w}_{2}-c_{y}\right)}+\left(1-\pi\left(y, \theta_{2}\right)\right) e^{-a\left(\underline{w}_{2}-c_{y}\right)}\right] \geq-e^{-a \bar{u}}
\end{array}\right. \\
& \left(P^{\text {flex }}\right)
\end{aligned}
$$

where incentive constraints (IC1),(IC2) and (IC3) ensure that, in state $\theta_{1}$, the agent does not want to deviate by, respectively, misreporting the state, modifying his action, or doing both. Incentive constraints (IC4), (IC5) and (IC6) ensure the same properties hold in state $\theta_{2}$. (PC) is the participation constraint.

We show in the next proposition that, at a solution to the above problem, only constraints (IC3), (IC4) and (PC) bind. We also derive properties of the optimal compensation scheme.

Proposition 1 Under Assumptions 1 and 2, there exists a flexible contract implementing action $x$ in $\theta_{1}$ and $y$ in $\theta_{2}$. The optimal contract implementing such a profile is obtained 
as solution of the following simplified problem:

$$
\begin{aligned}
\left.\max _{\bar{w}_{1}, \underline{w}_{1}, \bar{w}_{2}, \underline{w}_{2} \quad p[} \quad \pi\left(x, \theta_{1}\right)\left(\bar{R}-\bar{w}_{1}\right)+\left(1-\pi\left(x, \theta_{1}\right)\right)\left(\underline{R}-\underline{w}_{1}\right)\right] \\
+(1-p)\left[\pi\left(y, \theta_{2}\right)\left(\bar{R}-\bar{w}_{2}\right)+\left(1-\pi\left(y, \theta_{2}\right)\right)\left(\underline{R}-\underline{w}_{2}\right)\right]
\end{aligned}
$$

s.t. $(I C 3),(I C 4),(P C)$ holding as equalities and $\quad \bar{w}_{1} \geq \bar{w}_{2}, \bar{w}_{2} \geq \underline{w}_{2}$

and it exhibits the following properties:

$$
\bar{w}_{1} \geq \bar{w}_{2}>\underline{w}_{2} \geq \underline{w}_{1} .
$$

Recall that (IC3) refers to the 'joint deviation' in state $\theta_{1}$ (i.e., announcing instead the state is $\theta_{2}$ and choosing the less costly action $y$ rather than $x$ ), while (IC4) only concerns the mis-reporting deviation of announcing $\theta_{1}$ when the true state is $\theta_{2}$ (and still do action $y$, recommended in $\theta_{2}$ ). The private information about the state expands the agent's possible deviations and so imposes tighter constraints on the principal, as discussed in Remark 2 below.

Let $u\left(\theta_{1}\right)=-\pi\left(x, \theta_{1}\right) e^{-a\left(\bar{w}_{1}-c_{x}\right)}-\left(1-\pi\left(x, \theta_{1}\right)\right) e^{-a\left(\underline{w}_{1}-c_{x}\right)}$ denote the agent's expected utility at the optimal contract when state $\theta_{1}$ occurs; similarly, $u\left(\theta_{2}\right)=-\pi\left(y, \theta_{2}\right) e^{-a\left(\bar{w}_{2}-c_{y}\right)}-$ $\left(1-\pi\left(y, \theta_{2}\right)\right) e^{-a\left(\underline{w}_{2}-c_{y}\right)}$ is the utility when $\theta_{2}$ occurs. The properties shown in the above proposition that (IC3) is binding at an optimum and that $\bar{w}_{2}>\underline{w}_{2}$, together with the fact that $\pi\left(y, \theta_{1}\right)<\pi\left(y, \theta_{2}\right)$, yield the following important implication:

Corollary 1 At the optimal flexible contract, $u\left(\theta_{2}\right)>u\left(\theta_{1}\right)$.

Thus even though the cheaper action $y$ is implemented in state $\theta_{2}$ the optimal contract is characterized in that state by a wage that varies with the output realizations. At the same time, the expected utility of the net compensation paid to the agent is higher in state $\theta_{2}$ than in $\theta_{1}$. To understand these features, note that a fixed wage in state $\theta_{2}$ would be more attractive to a risk averse agent when the actual state is $\theta_{1}$ and hence a deviation consisting in misreporting the true state in $\theta_{1}$ and choosing action $y$ becomes more profitable. The variability of $w_{2}$ allows then to decrease the gains from such a deviation and hence to decrease the spread in $w_{1}$ needed to implement ${ }^{6} x$ in $\theta_{1}$. Similarly, the higher expected utility in state $\theta_{2}$ makes the misreporting deviation in that state less attractive.

\footnotetext{
${ }^{6}$ It can in fact be verified that (IC3), (IC4) and (PC) can all be satisfied as equality even with a constant level of $w_{2}$ - and hence with the same utility levels for the agent in state $\theta_{2}$ as in $\theta_{1}$ - but this is not optimal.
} 
Remark 2 To further understand the determinants of these properties of the optimal flexible contract, it is useful to compare them with those of the optimal contract obtained when the realization of $\theta$ is publicly observable while the agent's action is still not observable. The only incentive constraints which apply in this case - the contract still implements action $x$ in state $\theta_{1}$ and $y$ in $\theta_{2}$ - are (IC2), (IC5). The problem is thus clearly simpler and an explicit solution for the optimal compensation scheme can be derived. It is easy to verify ${ }^{7}$ that at an optimum in this case the only binding constraint is (IC2), there is a constant wage in state $\theta_{2}, \bar{w}_{2}=\underline{w}_{2}, \bar{w}_{1}>\underline{w}_{1}$ and full insurance across states, $u\left(\theta_{2}\right)=u\left(\theta_{1}\right){ }^{8}$ Thus the variability in $w_{2}$ and in the agent's utility levels across the realizations of $\theta$ we found in the optimal flexible contract (Proposition 1) is due to the need of addressing the additional incentive problems arising from the agent's private information over $\theta$. A lower variability in $w_{2}$ could only be achieved, as we already argued, at the cost of a higher variability of $w_{1}$.

Remark 3 On the last point above, it is useful to point out that the optimal contract obtained when $\theta$ is publicly observable is also optimal when $\theta$ is not observable but part ii) of Assumption 2 is replaced by the condition $\pi\left(x, \theta_{1}\right)>\pi\left(x, \theta_{2}\right)>\pi\left(y, \theta_{1}\right)>\pi\left(y, \theta_{2}\right)$, or by $\pi\left(x, \theta_{1}\right)>\pi\left(y, \theta_{1}\right)>\pi\left(x, \theta_{2}\right)>\pi\left(y, \theta_{2}\right)$. Under these specifications of the probabilities it is still true that $x$ is more productive than $y$ and, under iii), that the additional productivity of $x$ relative to $y$ is higher in state $\theta_{1}$, but now both actions $x$ and $y$ are more productive in state $\theta_{1}$ than in $\theta_{2}$. As a consequence, the mis-reporting deviation of announcing the state is $\theta_{2}$ when it is $\theta_{1}$ is less profitable: it can be verified that in this case $^{9}$ all the additional incentive constraints, (IC1, IC3, IC4, IC6) are satisfied at the contract discussed in Remark 2. In contrast, this is not true for the specification satisfying part ii) of Assumption 2, in which case (IC4) is violated, as well as for the other possible specifications consistent with part iii), where both $x$ and $y$ are more productive in state $\theta_{2}$ than $\theta_{1}$, in which case both (IC4) and (IC6) are violated.

7 For a formal statement of the claim and its proof, see the Online Appendix, available at http://www.eui.eu/Personal/Gottardi/.

${ }^{8}$ In the symmetric case where instead the agent's action is observable but $\theta$ is not, the only relevant constraints are (IC3), (IC6), and (PC). It is easy to verify that these constraints are satisfied if a constant wage $w_{1}=\bar{u}+c_{x}$ is paid to the agent when he announces $\theta_{1}$ and a constant wage $w_{2}=\bar{u}+c_{y}$ when he announces $\theta_{2}$. In this case the agent is fully insured, as his utility in state $\theta_{1}$ is equal to that in state $\theta_{2}$, and is independent of the output level. The action profile $x$ in $\theta_{1}$ and $y$ in $\theta_{2}$ can then be implemented at no extra cost for the principal.

${ }^{9}$ See the Online Appendix. 


\subsection{Rigid contracts}

The optimal rigid contract implementing a constant action $z \in\{x, y\}$ in every state is obtained as a solution of the following programme (note that the only constraint is given by $(\mathrm{PC})$, no incentive compatibility constraint appears here as the agent has no discretion over the choice of his action):

$$
\begin{aligned}
& \max _{\bar{w}_{1}, \underline{w}_{1}, \bar{w}_{2}, \underline{w}_{2}} \quad p\left[\pi\left(z, \theta_{1}\right)\left(\bar{R}-\bar{w}_{1}\right)+\left(1-\pi\left(z, \theta_{1}\right)\right)\left(\underline{R}-\underline{w}_{1}\right)\right] \\
& +(1-p)\left[\pi\left(z, \theta_{2}\right)\left(\bar{R}-\bar{w}_{2}\right)+\left(1-\pi\left(z, \theta_{2}\right)\right)\left(\underline{R}-\underline{w}_{2}\right)\right] \\
& (P C) \quad p\left[\pi\left(z, \theta_{1}\right) e^{-a\left(\bar{w}_{1}-c_{z}\right)}+\left(1-\pi\left(z, \theta_{1}\right)\right) e^{-a\left(\underline{w}_{1}-c_{z}\right)}\right]+ \\
& (1-p)\left[\pi\left(z, \theta_{2}\right) e^{-a\left(\bar{w}_{2}-c_{z}\right)}+\left(1-\pi\left(z, \theta_{2}\right)\right) e^{-a\left(\underline{w}_{2}-c_{z}\right)}\right]=e^{-a \bar{u}}
\end{aligned}
$$

Its solution is very simple in the present framework: the wage should be constant $\left(\bar{w}_{1}=\underline{w}_{1}=\bar{w}_{2}=\underline{w}_{2}=w_{z}\right)$, at the level determined by the participation constraint, thus equal to the expected cost of undertaking action $z .{ }^{10}$ In particular:

i) For the fixed $x$ contract, the compensation is $w_{x}=\bar{u}+c_{x}$, and expected profits are:

$$
\left[p \pi\left(x, \theta_{1}\right)+(1-p) \pi\left(x, \theta_{2}\right)\right] \bar{R}+\left[p\left(1-\pi\left(x, \theta_{1}\right)\right)+(1-p)\left(1-\pi\left(x, \theta_{2}\right)\right)\right] \underline{R}-\bar{u}-c_{x}
$$

ii) For the fixed $y$ contract, the compensation is $w_{y}=\bar{u}+c_{y}$, and profits are:

$$
\left[p \pi\left(y, \theta_{1}\right)+(1-p) \pi\left(y, \theta_{2}\right)\right] \bar{R}+\left[p\left(1-\pi\left(y, \theta_{1}\right)\right)+(1-p)\left(1-\pi\left(y, \theta_{2}\right)\right)\right] \underline{R}-\bar{u}-c_{y}
$$

\subsection{The choice between flexible and rigid contracts: the effect of risk aversion}

In this section we compare the expected profits of the principal at the optimal flexible contract with the expected profits at the rigid contracts. An important determinant of the agency cost of implementing a variable action profile is given by the agent's risk attitude (described, in the case of CARA preferences, by the single parameter $a$ ); in this comparison we focus then on the role played by this feature. As shown above, the compensation paid at the rigid contracts is a deterministic amount, whatever the agent's degree of risk aversion. In contrast, at the optimal flexible contract the compensation

\footnotetext{
${ }^{10}$ The agent is then fully insured. Since a constant action is undertaken, it would not help to make the payment contingent on the agent's announcement of $\theta$.
} 
varies both with $\theta$ and the output realizations, and hence the degree of risk aversion matters.

It is useful to consider first the extreme case where the agent is risk neutral, like the principal. In such a situation the optimal flexible contract is always preferable to the rigid ones: with this contract the principal can in fact attain the same level of profits as when all incentive compatibility constraints are ignored, hence agency costs are zero and the first best is achieved.

Proposition 2 When the agent is risk neutral the optimal flexible contract is first best optimal. The expected level of profits is $p\left[\pi\left(x, \theta_{1}\right) \bar{R}+\left(1-\pi\left(x, \theta_{1}\right)\right) \underline{R}\right]+(1-p)\left[\pi\left(y, \theta_{2}\right) \bar{R}+\right.$ $\left.\left(1-\pi\left(y, \theta_{2}\right)\right) \underline{R}\right]-\bar{u}-p c_{x}-(1-p) c_{y}$ and an optimal compensation ${ }^{11}$ is given by

$$
\begin{aligned}
& \bar{w}_{1}=\bar{u}+c_{x}+\frac{1-\pi\left(x, \theta_{1}\right)}{\pi\left(x, \theta_{1}\right)-\pi\left(y, \theta_{2}\right)} \Delta c \\
& \underline{w}_{1}=\bar{u}+c_{x}-\frac{\pi\left(x, \theta_{1}\right)}{\pi\left(x, \theta_{1}\right)-\pi\left(y, \theta_{2}\right)} \Delta c \\
& \bar{w}_{2}=\underline{w}_{2}=\bar{u}+c_{y}
\end{aligned}
$$

In contrast, when the agent is risk averse $(a>0)$ a risk premium must be paid to satisfy the incentive constraints, agency costs are then positive. In particular, when $a$ is sufficiently large (in particular, higher than the upper bound defined by Assumption $2(\mathrm{iv}))$ the variable action profile $(x, y)$ is no longer implementable and so rigid contracts are always preferable.

Besides the comparison of these extreme values of $a$, where the outcome is clear, we are also interested here in analysing the relative profitability of rigid and flexible contracts for intermediate values of $a$ and how it varies with small changes in the agents' degree of risk aversion. To this end we need to describe the effects of local changes in $a$ on the properties of the optimal flexible contract. This proves rather complex and no analytic result can be established. ${ }^{12}$ Indeed, when trying to disentangle the various effects of risk aversion, observe first that increasing $a$ makes the participation constraint, ceteris paribus, harder to satisfy: such constraint requires that the certainty equivalent of the lottery with outcomes $\bar{w}_{1}-c_{x}, \underline{w}_{1}-c_{x}, \bar{w}_{2}-c_{y}, \underline{w}_{2}-c_{y}$ is equal to $\bar{u}$, but the certainty equivalent of this lottery decreases with risk aversion. Consider then the incentive constraints which

\footnotetext{
${ }^{11}$ Note that this compensation scheme yields $u\left(\theta_{2}\right)=u\left(\theta_{1}\right)$.

${ }^{12}$ The difficulties faced in the comparative statics analysis with respect to risk aversion were also emphasized by Jullien, Salanié and Salanié (1999).
} 
are binding at an optimum solution, (IC3) and (IC4): each of them requires two distinct lotteries to have the same expected utility. For (IC4) we can say that the second lottery that is compared is always riskier than the first one. Hence increasing risk aversion loosens this constraint: i.e., if $a$ is increased while the compensation is kept constant, the constraint becomes slack. On the other hand, in the case of (IC3) we cannot rank, in terms of riskiness, the two lotteries that are compared. ${ }^{13}$ Hence, when $a$ increases, (PC) is harder to satisfy while (IC4) is easier, and the effect on (IC3) is unclear.

Given these difficulties in the analysis in the rest of this section we rely on the consideration of a numerical example, where the parameters describing the environment exhibit the following values: ${ }^{14}$

\begin{tabular}{|c|c|c|c|c|c|c|c|c|c|c|}
\hline$a$ & $p$ & $\bar{u}$ & $\bar{R}$ & $\underline{R}$ & $c_{x}$ & $c_{y}$ & $\pi\left(x, \theta_{1}\right)$ & $\pi\left(x, \theta_{2}\right)$ & $\pi\left(y, \theta_{1}\right)$ & $\pi\left(y, \theta_{2}\right)$ \\
\hline 1 & .5 & 1 & 10 & 5 & 1.5 & 1 & .8 & .45 & .2 & .4 \\
\hline
\end{tabular}

Table 1:

Figure 1 shows how the difference between the expected profits at the optimal flexible contract and the two rigid contracts changes with $a$ : this difference is monotonically decreasing. We see in particular that for low levels of risk aversion, the flexible contract is preferable to the two rigid contracts, but as $a$ increases the profit differential becomes progressively smaller and eventually, from $a \sim 1.6$ onwards in the situation considered, the rigid contract specifying task $x$ for the agent becomes optimal. We should stress that this pattern is robust to changes in the value of the parameters. Hence these numerical findings allow us to assert that agency costs are increasing with the agent's risk aversion ${ }^{15}$. Hence we conclude that agency costs are increasing and the advantages of delegation are decreasing in the agent's degree of risk aversion.

\section{INCLUDE FIGURE 1 ABOUT HERE}

\footnotetext{
${ }^{13}$ These are $\left(\bar{w}_{1}-c_{x}, \underline{w}_{1}-c_{x}\right)$, with probabilities $\pi\left(x, \theta_{1}\right), 1-\pi\left(x, \theta_{1}\right)$, and $\left(\bar{w}_{2}-c_{y}, \underline{w}_{2}-c_{y}\right)$, with probabilities $\left(\pi\left(y, \theta_{1}\right), 1-\pi\left(y, \theta_{1}\right)\right)$. We know that $\underline{w}_{1}-c_{x}$ is the smallest outcome but we do not know how to rank $\bar{w}_{1}-c_{x}$ versus $\bar{w}_{2}-c_{y}$ and the attached probabilities are not the same. Thus, the effect of changing risk aversion on this constraint is ambiguous.

${ }^{14}$ The values of the parameters have been chosen so as to satisfy the restrictions imposed by Assumption 2 but are otherwise arbitrary. As commented below in the text, we considered however also several other possible specifications of the parameters satisfying this restriction.

${ }^{15}$ A similar pattern also obtains when the realization of $\theta$ is commonly observed: increasing risk aversion makes the rigid contracts more attractive relative to the flexible ones. The profits of the flexible contract when $\theta$ is observable are strictly higher than when $\theta$ is only privately observed, and the difference is increasing in risk aversion.
} 
We also find ${ }^{16}$ that, in this environment, the wage spread in state $\theta_{1}$ (i.e., the difference between the compensation paid for the high and low output realizations, $\left.\bar{w}_{1}-\underline{w}_{1}\right)$ is first decreasing and then increasing in $a$ while the spread in $\theta_{2}$ is always increasing in $a$. The utility differential across states also varies non monotonically with $a$, first increasing and then decreasing.

Summing up the main findings obtained so far, we have seen that, somewhat counterintuitively, the optimal flexible contract entails some variability of the wage in the state in which the less costly (and less productive) action is implemented. This variability is needed to prevent the agent from misreporting the state when in the good state. There is also some variability in the utility across states. Quite intuitively, these variability in the optimal flexible contract makes it less attractive the higher the risk aversion of the agent.

\section{The choice of delegation with imprecise beliefs}

We examine now the case where, at the time in which the contract is written, the information available to the parties concerning the likelihood of the various events is not precise enough for them to have a sharp probability belief, i.e., the situation faced by the parties is sufficiently new that past data cannot be used to pin down probabilities.

We thus assume in this section that there is a set of probability distributions over $\left\{\theta_{1}, \theta_{2}\right\}$, described by an interval $[\underline{p}, \bar{p}]$ of values for the probability $p$ that event $\theta_{1}$ occurs. This set represents the probability beliefs consistent with the available information (precise information corresponds to a singleton set, $\underline{p}=\bar{p})$. Similarly, for each $z \in\{x, y\}$ and $\theta \in\left\{\theta_{1}, \theta_{2}\right\}$ there is an interval $[\underline{\pi}(z, \theta), \bar{\pi}(z, \theta)]$ of possible probabilities that the output level is $\bar{R}$ when event $\theta$ occurs and the action chosen is $z$. These sets of distributions are to be interpreted as summing up, for both parties, all the information commonly available on the uncertainty they face.

\subsection{Imprecision, imprecision aversion and the contracting prob- lem}

We need a tractable model of decision under uncertainty in such situations, that allows for a parametrization of individuals' attitude towards ambiguity (or imprecision, as we call it) and hence for comparative static exercises. To this end we use the model developed

\footnotetext{
${ }^{16}$ See the Online Appendix for further details.
} 
by Gajdos et al. (2008). In the case of interest here, given the simple two-state structure of the uncertainty, this model is particularly simple. It says that the preferences of the decision maker can be represented by a convex combination, with respective weights $\alpha$, $1-\alpha$, of the minimal expected utility (with respect to all possible distributions in the specified intervals) and the expected utility with respect to a central probability (the center of the intervals).

More precisely, when the probability of the first state $\left(\theta_{1}\right)$ lies between a lower bound $\underline{p}$ and an upper bound $\bar{p}$, with $\hat{p}=\frac{p+\bar{p}}{2}$ being the 'central' probability, the utility of an action $f$ is described by $\alpha \min _{p \in[\underline{p}, \bar{p}]} E_{p} u(f)+(1-\alpha) E_{\hat{p}} u(f)$, where $E_{p} u(f)$ is the expected utility over the consequences of action $f$ evaluated with beliefs $p$. Gajdos et al. (2008) provide an axiomatization of this criterion, showing that the weight $\alpha$ placed on the first term can be interpreted as a coefficient reflecting the decision maker's aversion to the imprecision represented by the interval $[\underline{p}, \bar{p}]$. The case $\alpha=0$ reflects imprecision neutrality: the decision maker acts as if he were an expected utility maximizer with respect to the central probability in each interval, while $\alpha=1$ reflects extreme imprecision aversion, with the decision maker putting all the weight on the least favorable prior.

Remark 4 The model described above can be recast as a 'maxmin' model in the sense of Gilboa and Schmeidler (1989). Just consider the minimum expected utility taken over the set of probabilities that lie in the subinterval $\left[\hat{p}-\alpha \frac{\underline{p}+\bar{p}}{2}, \hat{p}+\alpha \frac{\underline{p}+\bar{p}}{2}\right]$ of $[\underline{p}, \bar{p}]$. This subinterval is obtained simply by 'shrinking' the original interval describing the set of probabilities symmetrically towards its center, at a rate equal to the decision maker's degree of imprecision aversion $\alpha$.

The difference between the models thus does not lie in the functional form per se, rather in its interpretation. Here, following Gajdos et al. (2008), we interpret $[\underline{p}, \bar{p}]$ as an objective set of probabilistic models which reflects the imprecision in the (common) information available to the parties. The decision maker then shrinks this set as a result of his (subjective) aversion to the imprecision (the principal, being imprecision neutral, shrinks this set in a maximal way and behaves as an expected utility maximizer with respect to the center of the interval.) In Gilboa and Schmeidler (1989), the set of priors $\left[\hat{p}-\alpha \frac{\underline{\underline{p}+\bar{p}}}{2}, \hat{p}+\alpha \frac{\underline{\underline{p}+\bar{p}}}{2}\right]$ is interpreted as the decision maker's subjective perception of the ambiguity in the problem. 
Remark 5 Because of our two-state setting, the model above is also equivalent ${ }^{17}$ to an $\alpha$ maxmin model: $\alpha \min _{p \in[\underline{p}, \bar{p}]} E_{p} u(f)+(1-\alpha) E_{\hat{p}} u(f) \propto \frac{\alpha+1}{2} \min _{p \in[\underline{p}, \bar{p}]} E_{p} u(f)+\left(1-\frac{\alpha}{2}\right) \max _{p \in[\underline{p}, \bar{p}]} E_{p} u(f)$, where $[\underline{p}, \bar{p}]$ is the ambiguity in the problem and $\beta=(\alpha+1) / 2$ is a measure of the decision maker's aversion to such ambiguity (with $\beta=1$ representing extreme aversion to ambiguity and $\beta=1 / 2$ representing ambiguity neutrality). Such a model has been axiomatized by Jaffray (1989) in a setup with objective information and Ghirardato et al. (2004) in a purely subjective setup. Hence, the results below can also be extended to these models.

In this section we assume that both parties are risk neutral. We furthermore assume that the principal is imprecision neutral. He therefore acts as an expected profit maximizer, with respect to the central probability level. The agent, on the other hand, is characterized by his degree $\alpha$ of imprecision aversion.

The principal's objective function - when implementing the flexible contract - is then to maximize

$\hat{p}\left[\hat{\pi}\left(x, \theta_{1}\right)\left(\bar{R}-\bar{w}_{1}\right)+\left(1-\hat{\pi}\left(x, \theta_{1}\right)\right)\left(\underline{R}-\underline{w}_{1}\right)\right]+(1-\hat{p})\left[\hat{\pi}\left(y, \theta_{2}\right)\left(\bar{R}-\bar{w}_{2}\right)+\left(1-\hat{\pi}\left(y, \theta_{2}\right)\right)\left(\underline{R}-\underline{w}_{2}\right)\right]$,

where we use a ^ to denote the 'central probabilities,' i.e., $\hat{p}=\frac{\underline{p}+\bar{p}}{2}, \hat{\pi}(z, \theta)=\frac{\frac{\pi(z, \theta)+\bar{\pi}(z, \theta)}{2}}{2}$ for each $z \in\{x, y\}$ and $\theta \in\left\{\theta_{1}, \theta_{2}\right\}$.

Consider next the agent's incentive constraints. His utility in event $\theta_{1}$ when action $x$ is exerted is given by

$\alpha \min _{\pi\left(x, \theta_{1}\right) \in\left[\pi\left(x\left(x, \theta_{1}\right), \bar{\pi}\left(x, \theta_{1}\right)\right]\right.}\left\{\pi\left(x, \theta_{1}\right) \bar{w}_{1}+\left(1-\pi\left(x, \theta_{1}\right)\right) \underline{w}_{1}\right\}+(1-\alpha)\left[\hat{\pi}\left(x, \theta_{1}\right) \bar{w}_{1}+\left(1-\hat{\pi}\left(x, \theta_{1}\right)\right) \underline{w}_{1}\right]$

As explained above, this can also be expressed as

$$
\min _{\pi \in\left[\hat{\pi}\left(x, \theta_{1}\right)-\alpha\left(x, \theta_{1}\right), \hat{\pi}\left(x, \theta_{1}\right)+\alpha\left(x, \theta_{1}\right)\right]}\left\{\pi \bar{w}_{1}+(1-\pi) \underline{w}_{1}\right\},
$$

where $\alpha\left(x, \theta_{1}\right)=\alpha \frac{\bar{\pi}\left(x, \theta_{1}\right)-\underline{\pi}\left(x, \theta_{1}\right)}{2}$.

To simplify notation, denote by $I\left(x, \theta_{1}\right)$ the interval $\left[\hat{\pi}\left(x, \theta_{1}\right)-\alpha\left(x, \theta_{1}\right), \hat{\pi}\left(x, \theta_{1}\right)+\right.$ $\left.\alpha\left(x, \theta_{1}\right)\right]$. Call 'induced belief' the probability that the agent uses to evaluate the wage profile $\bar{w}_{1}, \underline{w}_{1}$, that is the element of $I\left(x, \theta_{1}\right)$ which minimizes $\pi \bar{w}_{1}+(1-\pi) \underline{w}_{1}$ : this is the most pessimistic belief in $I\left(x, \theta_{1}\right)$ to evaluate the given wage profile. This belief will depend on the ordering of the wages across output realizations (i.e., whether $\bar{w}_{1} \gtrless \underline{w}_{1}$ ) and on the imprecision aversion parameter $\alpha$. The principal, by choosing different profiles

\footnotetext{
${ }^{17}$ Ahn, Choi, Gale and Kariv (2014) also note this formal analogy.
} 
of wages can induce different beliefs for the agent. An exception is the case where the principal chooses to fully insure the agent in state $\theta_{1}\left(\bar{w}_{1}=\underline{w}_{1}\right)$, as in that situation the beliefs of the agent are not pinned down uniquely, since all beliefs provide the same constant evaluation of the wage profile.

Using similar notation for the agent's utility in event $\theta_{2}$, and when action $y$ is chosen in $\theta_{1}$ and $\theta_{2}$, the expressions of the incentive constraints, analogous to those in $\left(P^{f l e x}\right)$, are as follows:

$$
\begin{cases}\left(I C 1^{*}\right) \min _{\pi \in I\left(x, \theta_{1}\right)}\left[\pi \bar{w}_{1}+(1-\pi) \underline{w}_{1}\right] & \geq \min _{\pi \in I\left(x, \theta_{1}\right)}\left[\pi \bar{w}_{2}+(1-\pi) \underline{w}_{2}\right] \\ \left(I C 2^{*}\right) \min _{\pi \in I\left(x, \theta_{1}\right)}\left[\pi \bar{w}_{1}+(1-\pi) \underline{w}_{1}\right]-c_{x} \geq \min _{\pi \in I\left(y, \theta_{1}\right)}\left[\pi \bar{w}_{1}+(1-\pi) \underline{w}_{1}\right]-c_{y} \\ \left(I C 3^{*}\right) \min _{\pi \in I\left(x, \theta_{1}\right)}\left[\pi \bar{w}_{1}+(1-\pi) \underline{w}_{1}\right]-c_{x} \geq \min _{\pi \in I\left(y, \theta_{1}\right)}\left[\pi \bar{w}_{2}+(1-\pi) \underline{w}_{2}\right]-c_{y} \\ \left(I C 4^{*}\right) \min _{\pi \in I\left(y, \theta_{2}\right)}\left[\pi \bar{w}_{2}+(1-\pi) \underline{w}_{2}\right] & \geq \min _{\pi \in I\left(y, \theta_{2}\right)}\left[\pi \bar{w}_{1}+(1-\pi) \underline{w}_{1}\right] \\ \left(I C 5^{*}\right) \min _{\pi \in I\left(y, \theta_{2}\right)}\left[\pi \bar{w}_{2}+(1-\pi) \underline{w}_{2}\right]-c_{y} \geq \min _{\pi \in I\left(x, \theta_{2}\right)}\left[\pi \bar{w}_{2}+(1-\pi) \underline{w}_{2}\right]-c_{x} \\ \left(I C 6^{*}\right) \min _{\pi \in I\left(y, \theta_{2}\right)}\left[\pi \bar{w}_{2}+(1-\pi) \underline{w}_{2}\right]-c_{y} \geq \min _{\pi \in I\left(x, \theta_{2}\right)}\left[\pi \bar{w}_{1}+(1-\pi) \underline{w}_{1}\right]-c_{x}\end{cases}
$$

The participation constraint $\left(P C^{*}\right)$ takes then the following form:

$\min _{p \in[\hat{p}-\alpha(p), \hat{p}+\alpha(p)]}\left\{p\left[\min _{\pi \in I\left(x, \theta_{1}\right)}\left[\pi \bar{w}_{1}+(1-\pi) \underline{w}_{1}\right]-c_{x}\right]+(1-p)\left[\min _{\pi \in I\left(y, \theta_{2}\right)}\left[\pi \bar{w}_{2}+(1-\pi) \underline{w}_{2}\right]-c_{y}\right]\right\} \geq \bar{u}$

where $\alpha(p)=\alpha \frac{\bar{p}-\underline{\underline{p}}}{2}$. As a solution to all the above minimization problems, we obtain the system of beliefs induced by the wage profile considered for all states and all possible action choices.

Finally, it is convenient to reformulate part ii) of Assumption 2 in the present framework as follows:

$$
\begin{gathered}
\text { for all } \pi\left(x, \theta_{1}\right) \in I\left(x, \theta_{1}\right), \pi\left(x, \theta_{2}\right) \in I\left(x, \theta_{2}\right), \pi\left(y, \theta_{2}\right) \in I\left(y, \theta_{2}\right), \pi\left(y, \theta_{1}\right) \in I\left(y, \theta_{1}\right), \\
\text { we have } \pi\left(x, \theta_{1}\right)>\pi\left(x, \theta_{2}\right)>\pi\left(y, \theta_{2}\right)>\pi\left(y, \theta_{1}\right)
\end{gathered}
$$

This ensures that there is no overlap in the intervals $I(.,$.$) , and thus that the induced$ beliefs (no matter what they are) respect the ordering we imposed in Assumption 2(ii) where these beliefs were assumed to be precise, single probability distributions ${ }^{18}$. This is now a joint assumption on $\alpha$, the imprecision aversion of the agent, and the 'amount of imprecision,' captured by the width of the probability intervals $[\underline{\pi}(z, \theta), \bar{\pi}(z, \theta)]$.

Recalling the discussion in Remark 4, as it should be clear from the specification of the objective function and the constraints in (2), the analysis carries over to the maxmin

\footnotetext{
${ }^{18}$ We refer to Section 2 and Remark 3 for a discussion justifying the focus on beliefs satisfying this condition.
} 
expected utility model of Gilboa and Schmeidler (1989), with the intervals I(.) taken as given (rather than derived from some underlying objective function and some level of imprecision aversion). The value added by the model of Gajdos et al. (2008) is the possibility of decomposing the ingredients entering the construction of the intervals $\mathrm{I}(.$.$) ,$ and to distinguish between the 'objective' (i.e., intersubjective) measure of imprecision and the subjective parameter of aversion towards imprecision.

\subsection{Optimal contract}

In this section we characterize the optimal flexible contract under imprecision aversion and discuss how it differs from the optimal contract under risk aversion.

We provide first a partial characterization of the optimal flexible contract in the present environment. It is useful to start by conjecturing that the optimal flexible contract takes the following form:

$$
\begin{aligned}
& \bar{w}_{1}=\bar{u}+c_{x}+\frac{1-\hat{\pi}\left(x, \theta_{1}\right)-\alpha\left(x, \theta_{1}\right)}{\hat{\pi}\left(x, \theta_{1}\right)-\alpha\left(x, \theta_{1}\right)-\hat{\pi}\left(y, \theta_{2}\right)+\alpha\left(y, \theta_{2}\right)} \Delta c \\
& \underline{w}_{1}=\bar{u}+c_{x}-\frac{\hat{\pi}\left(x, \theta_{1}\right)-\alpha\left(x, \theta_{1}\right)}{\hat{\pi}\left(x, \theta_{1}\right)-\alpha\left(x, \theta_{1}\right)-\hat{\pi}\left(y, \theta_{2}\right)+\alpha\left(y, \theta_{2}\right)} \Delta c \\
& \bar{w}_{2}=\underline{w}_{2}=\bar{u}+c_{y}
\end{aligned}
$$

This is the natural extension to the present environment of the contract which, as we saw in Proposition 2, allows to attain the first best in the risk neutral and imprecision neutral case. Note however that in the presence of imprecision aversion this contract may no longer be first-best optimal since it features variability of the wage in state $\theta_{1}$ and this, as we will see in what follows, may entail a loss in social surplus, even if the agent is risk neutral.

To determine whether and when the wage profile in (3) is indeed an optimal flexible contract, note first that, at such contract, the incentive constraints $\left(I C 3^{*}\right)$ and $\left(I C 4^{*}\right)$ in (2) are binding, while the others are slack. The participation constraint $\left(P C^{*}\right)$ is also binding. We investigate then whether local deviations from it $\left(d \bar{w}_{1}, d \underline{w}_{1}, d \bar{w}_{2}, d \underline{w}_{2}\right)$, satisfying $\left(I C 3^{*}\right),\left(I C 4^{*}\right)$ and $\left(P C^{*}\right)$ as equality ${ }^{19}$, can increase the expected level of the principal's profit. We outline in what follows the main steps of the argument, referring to the Appendix for further details.

\footnotetext{
${ }^{19}$ Given the linearity of the constraints in $w$, this is w.l.o.g..
} 
Use the system given by the three binding, linear constraints to solve for $d \bar{w}_{1}, d \bar{w}_{2}$, and $d \underline{w}_{2}$ as a function of $d \underline{w}_{1}$. At the contract in (3), the beliefs regarding $\theta$ are indeterminate since the utility is the same in the two $\theta$ states, while this may not be true for the deviations considered. We need to distinguish deviations where $d \underline{w}_{1}>0$ and those where $d \underline{w}_{1}<0$. If $d \underline{w}_{1}>0$, it can be easily verified that $d \bar{w}_{1}<0, d \bar{w}_{2}>0$, and $d \underline{w}_{2}<0$, while if $d \underline{w}_{1}<0$, the opposite signs obtain. The induced beliefs are uniquely pinned down by the two deviations, in different -opposite--ways since the utilities change in opposite ways in these two cases.

We can now write the change in the profit of the principal as a function of $d \underline{w}_{1}$ alone for each of the two types of deviations described above. ${ }^{20}$ It is immediate to verify that the change in the profit is always negative when $d \underline{w}_{1}<0$. Hence this type of deviation is never profit-improving for the principal. On the other hand, we show in the Appendix that the other type of deviation, $d \underline{w}_{1}>0$ is also not profitable only when the following condition is satisfied:

$$
\begin{aligned}
& 1>\frac{\hat{p} \hat{\pi}\left(x, \theta_{1}\right)+(1-\hat{p}) \hat{\pi}\left(y, \theta_{2}\right)}{\left[(\hat{p}+\alpha(p))\left(\hat{\pi}\left(x, \theta_{1}\right)-\alpha\left(x, \theta_{1}\right)\right)+(1-\hat{p}-\alpha(p))\left(\hat{\pi}\left(y, \theta_{2}\right)-\alpha\left(y, \theta_{2}\right)\right)\right]} \\
& +(1-\hat{p}) \frac{\left[\hat{\pi}\left(x, \theta_{1}\right)-\alpha\left(x, \theta_{1}\right)-\hat{\pi}\left(y, \theta_{1}\right)+\alpha\left(y, \theta_{1}\right)\right] \alpha\left(y, \theta_{2}\right)}{\left[(\hat{p}+\alpha(p))\left(\hat{\pi}\left(x, \theta_{1}\right)-\alpha\left(x, \theta_{1}\right)\right)+(1-\hat{p}-\alpha(p))\left(\hat{\pi}\left(y, \theta_{2}\right)-\alpha\left(y, \theta_{2}\right)\right)\right]\left[\hat{\pi}\left(y, \theta_{1}\right)-\alpha\left(y, \theta_{1}\right)-\hat{\pi}\left(y, \theta_{2}\right)+\alpha\left(y, \theta_{2}\right)\right]}
\end{aligned}
$$

Hence when this condition holds, the optimal flexible contract is given by (3).

It is easy to verify that condition (4) is always satisfied when $\alpha\left(x, \theta_{1}\right)=0$, that is when there is no imprecision (as measured by the width of the interval $\left[\underline{\pi}\left(x, \theta_{1}\right), \bar{\pi}\left(x, \theta_{1}\right)\right]$ ) regarding the outcome of action $x$ in state $\theta_{1}$. In this situation, where the outcome of action $x$ in state $\theta_{1}$ is precisely assessed, the agent's utility for the compensation contract is the same as if the agent were not only risk but also imprecision neutral. Since the agent has no imprecision regarding the consequences of action $x$ in $\theta_{1}$, the variability in the wage in state $\theta_{1}$ - necessary to implement $x$ - entails no cost, in terms of lower utility for the agent which would need to be compensated by the principal, while the wage in (3) provides full insurance to the agent in state $\theta_{2}$ and across states $\theta_{1}$ and $\theta_{2}$, and hence no other cost for the agent. The contract in (3) achieves thus the first best and is the overall optimal contract, dominating all rigid contracts.

The same property clearly holds in an open interval around the point $\alpha\left(x, \theta_{1}\right)=0$, and hence (4) is satisfied for an open set of values of the parameters describing the economy.

\footnotetext{
${ }^{20}$ Note that, given the linearity of the utility function, this local approach is enough to draw global conclusions.
} 
In particular, when there is little imprecision on the outcome of action $x$ in state $\theta_{1}$, the utility loss for the agent due to the wage variability in that state is sufficiently small for the principal so that fully insuring the agent along the other dimension of the uncertainty remains optimal. In addition, the contract in (3) is still the optimal flexible contract, dominating the rigid contracts, even though it is no longer first best and there is now a positive loss in social surplus.

In contrast, when $\alpha(p)=\alpha\left(y, \theta_{2}\right)=0$ while $\alpha\left(x, \theta_{1}\right)$ is strictly positive and not too small, condition (4) does not hold and so the wage in (3) is not the optimal flexible contract. Recall that $\alpha(p)=\alpha\left(y, \theta_{2}\right)=0$ means there is no imprecision on $p$ - i.e., there is a known probability of the occurrence of $\theta_{1}, \theta_{2}$ - nor any imprecision regarding the probability of success in state $\theta_{2}$ when $y$ is undertaken. In contrast, $\alpha\left(x, \theta_{1}\right)>0$ means that there is imprecision regarding the probability of success in state $\theta_{1}$. In this case a reduction, with respect to the situation in (3), in the wage volatility in state $\theta_{1}$ (as in the considered deviation with $d \underline{w}_{1}>0$ ) allows to increase profits, even though the wage volatility in state $\theta_{2}$ increases. When $\alpha\left(x, \theta_{1}\right)>0$ the agent, when evaluating the wages in (3), uses in fact different beliefs in state $\theta_{1}$ from those of the principal, and this leads to a decrease in the surplus to be shared in that state. On the other hand, given that there is no imprecision on $p$ nor about the probability of success in state $\theta_{2}$ when $y$ is undertaken, increasing the wage variability in state $\theta_{2}$ and across states is of little consequence, since the agent evaluates his compensation as if risk neutral and imprecision neutral in these dimensions. The combination of these two facts explain why (3) is not optimal in this configuration. Clearly it is not possible to bring down to zero the volatility of wages in $\theta_{1}$, as some variability in this state is still required to implement action $x$ in that state.

Our findings are summarized in the next:

Proposition 3 When the parameter values describing the environment satisfy condition (4), the optimal flexible contract is given by (3). Otherwise, when (4) is violated, the optimal flexible contract is characterized by $\bar{w}_{2}>\underline{w}_{2}$ and by a lower variability than in (3) of the wages paid in state $\theta_{1}$.

The conclusion we draw from this analysis is that the optimal flexible contract under imprecision aversion (and risk neutrality) qualitatively differs from the optimal one under risk aversion (and imprecision neutrality). Under risk aversion only it is never optimal to provide full insurance to the agent across different output realizations in state $\theta_{2}$ as well 
as across states $\theta_{1}$ and $\theta_{2}$, while under imprecision aversion this is optimal for an open set of parameter values. This difference can be explained by the different channels through which an agency cost appears in these two (polar) cases, as we explore next.

\subsection{Differences in beliefs and the source of agency costs}

In this section we establish more formally the property mentioned in the previous section that the variability of the agent's compensation across different realizations of the uncertainty entails some costs in terms of lower social surplus. The cost of the wage variability comes from the fact that the induced beliefs of the agent change with the wage profile, and this 'pessimism' needs to be compensated by the principal. This provides a new channel via which the variability in the wage profile affects the agent's utility and hence his choices, as now the ranking of the payoffs across states matters to determine the induced beliefs, while this was irrelevant in the risk aversion case. Although this agency cost could be expected, it is worth noticing that fixing different beliefs for the agent and the principal would lead to 'speculative gains' that are absent in the present setup.

Let us start from the full insurance contract, that is the optimal contract absent any informational asymmetries. This contract insures the worker within each state $\theta$ as well as across states. It has $\bar{w}_{1}=\underline{w}_{1}=\bar{u}+c_{x}$ and $\bar{w}_{2}=\underline{w}_{2}=\bar{u}+c_{y}$. We show in what follows that any deviation $\left(d \bar{w}_{1}, d \underline{w}_{1}, d \bar{w}_{2}, d \underline{w_{2}}\right)$ from this contract which satisfies the participation constraint decreases the principal's profits when imprecision is not degenerate.

The change in expected cost for the principal is equal to

$$
\hat{p}\left[\hat{\pi}\left(x, \theta_{1}\right) d \bar{w}_{1}+\left(1-\hat{\pi}\left(x, \theta_{1}\right)\right) d \underline{w}_{1}\right]+\left(\hat{\pi}\left(y, \theta_{2}\right) d \bar{w}_{2}+\left(1-\hat{\pi}\left(y, \theta_{2}\right)\right)\right) d \underline{w}_{2} .
$$

Consider then the following deviation from the full insurance contract: $d \bar{w}_{1}>0, d \underline{w}_{1}<0$, $d \bar{w}_{2}>0$ and $d \underline{w_{2}}<0$ so that

$$
\begin{aligned}
& \left(\hat{\pi}\left(y, \theta_{2}\right)-\alpha\left(y, \theta_{2}\right)\right) d \bar{w}_{2}+\left(1-\hat{\pi}\left(y, \theta_{2}\right)+\alpha\left(y, \theta_{2}\right)\right) d \underline{w}_{2}> \\
& \left(\hat{\pi}\left(x, \theta_{1}\right)-\alpha\left(x, \theta_{1}\right)\right) d \bar{w}_{1}+\left(1-\hat{\pi}\left(x, \theta_{1}\right)+\alpha\left(x, \theta_{1}\right)\right) d \underline{w}_{1}
\end{aligned}
$$

i.e., the agent is now better off in state $\theta_{2}$ than in $\theta_{1}$. As a consequence, the agent now evaluates the occurrence of $\theta_{1}$ with the least favorable distribution, i.e., $\hat{p}+\alpha(p)$. Similarly, he uses $\hat{\pi}\left(x, \theta_{1}\right)-\alpha\left(x, \theta_{1}\right)$ to evaluate, within state $\theta_{1}$, the probability of $\bar{R}$, conditionally on doing action $x$, and $\hat{\pi}\left(y, \theta_{2}\right)-\alpha\left(y, \theta_{2}\right)$ to evaluate, within state $\theta_{2}$ the probability of $\bar{R}$, conditionally on doing action $y$. Having pinned down the beliefs induced 
by this deviation, we can compute the effect of this change in compensation on the agent's participation constraint. Differentiating the expression of this constraint in $\left(\mathrm{PC}^{\star}\right)$, using these beliefs, yields:

$$
\begin{aligned}
& \hat{p}\left[\hat{\pi}\left(x, \theta_{1}\right) d \bar{w}_{1}+\left(1-\hat{\pi}\left(x, \theta_{1}\right)\right) d \underline{w}_{1}\right]+(1-\hat{p})\left[\hat{\pi}\left(y, \theta_{2}\right) d \bar{w}_{2}+\left(1-\hat{\pi}\left(y, \theta_{2}\right)\right) d \underline{w}_{2}\right]+ \\
& {\left[\hat{p} \alpha\left(x, \theta_{1}\right)\left(d \underline{w}_{1}-d \bar{w}_{1}\right)+(1-\hat{p}) \alpha\left(y, \theta_{2}\right)\left(d \underline{w}_{2}-d \bar{w}_{2}\right)\right]+} \\
& \alpha(p)\left[\left(\hat{\pi}\left(x, \theta_{1}\right)-\alpha\left(x, \theta_{1}\right)\right) d \bar{w}_{1}+\left(1-\hat{\pi}\left(x, \theta_{1}\right)+\alpha\left(x, \theta_{1}\right)\right) d \underline{w}_{1}-\right. \\
& \left.\quad\left(\hat{\pi}\left(y, \theta_{2}\right)-\alpha\left(y, \theta_{2}\right)\right) d \bar{w}_{2}-\left(1-\hat{\pi}\left(y, \theta_{2}\right)+\alpha\left(y, \theta_{2}\right)\right) d \underline{w}_{2}\right] \geq 0
\end{aligned}
$$

The term in the first line equals, as we saw above, the change in the wage cost for the principal. The other two terms reflect the change in the agent's utility due to the change in his induced beliefs, which in turn reflect the effect of imprecision and imprecision aversion: if in fact $\alpha\left(x, \theta_{1}\right)=\alpha\left(y, \theta_{2}\right)=\alpha(p)=0$ (which occurs, for instance, if $\alpha=0$; that is, if the agent is imprecision neutral), or if there is altogether no imprecision (i.e., information is sufficiently precise so that the set of distributions describing the situation is reduced to a singleton), the terms appearing in the second to the fourth line in (5) are all zero. When imprecision matters, the term appearing on the second line is negative, given the signs of the change in the agent's wage that is considered. The last term, on the third and fourth line, is negative as well since this change implies that the utility in state $\theta_{2}$ is higher than in state $\theta_{1}$. Hence, for the participation constraint to still hold ((5) to be satisfied) it has to be the case when imprecision matters) that the term on the first line is positive, that is, that the principal's profits decrease as a result of the change in wage considered.

The deviation contemplated above generates a higher utility level in state $\theta_{2}$ than in $\theta_{1}$ as well as, in each $\theta$ state, for the high income realization. This pins down the induced beliefs that appear in the agent's participation constraint. The same type of reasoning can be applied for any other deviation from the full insurance contract: the induced beliefs will be different but expected costs increase anyway. Since we know that a constant level of wages in state $\theta_{1}$ violates incentive compatibility and hence some variability in wages is needed, we therefore conclude that there exists an agency cost. ${ }^{21}$

This cost is due to the pessimistic beliefs on the agent's side, which tend to underweigh - compared to the principal's beliefs - the realization of the good outcome $\bar{R}$. These beliefs are induced by the variability in wages in state $\theta_{1}$, necessary to lead the agent to choose

\footnotetext{
${ }^{21}$ This cost could be zero in some special cases.
} 
action $x$ in that state. This lower valuation by the agent of the compensation received forces the principal to raise it, compared to what he would do absent any informational asymmetries, in order to fulfill the participation constraint.

What this analysis also shows is that, in line with the no trade results present in the literature on ambiguity aversion (see, e.g., Billot et al. (2000), Strzalecki and Werner (2011)) it is never optimal to induce different beliefs between the agent and the principal, unless it is required to do so in order to satisfy the incentive constraints. And in this setting such difference in beliefs never increases the surplus to be split between the two parties. This is in stark contrast with a situation in which the principal and the agent have fixed and different probabilistic beliefs that do not depend on the contract considered.

To better understand the role played by this heterogeneity in beliefs between principal and agent induced by imprecision aversion, it is useful to examine the case where the beliefs of principal and agent are fixed at this induced level. Consider in particular the contract described in (3): the principal uses beliefs $\hat{\pi}\left(x, \theta_{1}\right)$ while the agent uses $\bar{\pi}\left(x, \theta_{1}\right)$. On the other hand, the beliefs of the agent over $\theta_{1}$ and on $\bar{R}$ conditionally on being in state $\theta_{2}$ and on doing action $y$ are not pinned down and could be set equal to those used by the principal, $\hat{p}$ and $\hat{\pi}\left(y, \theta_{2}\right)$. However when the agent and the principal have exogenously fixed beliefs set at this level $\left(\bar{\pi}\left(x, \theta_{1}\right), \hat{p}, \hat{\pi}\left(y, \theta_{2}\right)\right.$ for the agent and $\hat{\pi}\left(x, \theta_{1}\right), \hat{p}, \hat{\pi}\left(y, \theta_{2}\right)$ for the principal), the contract considered is never optimal: a higher level of expected profits can in fact be attained by reducing the volatility of the payment in the $\theta_{1}$ state and increasing that in $\theta_{2}$ and across the $\theta$ states. This stands in stark contrast to the imprecision aversion case, where the contract described is optimal when (4) holds. The reason is precisely because the deviation described above would induce a change in the agent's beliefs which would make the deviation no longer profitable.

\subsection{Flexible vs rigid contracts under imprecision aversion}

We can then again compare the optimal flexible contract to the rigid contracts. In particular, we analyze how the relative profitability of the two varies with respect to the parameters describing the imprecision aversion $(\alpha)$ and the agents' imprecision -as captured by the width of the intervals describing the possible probability beliefs.

Whenever the optimal flexible contract is given by (3), it is fairly easy to verify ${ }^{22}$ that expected profits are strictly decreasing in $\alpha$ as well as in the agents' imprecision. Since

\footnotetext{
${ }^{22}$ Details are in the Appendix.
} 
expected profits at the optimal rigid contracts are independent of $\alpha$ we obtain:

Corollary 2 Under (4) the relative profitability of flexible over rigid contracts is always decreasing in the agent's degree of imprecision aversion $\alpha$ as well as in the imprecision of the return to action $x$ in state $\theta_{1}$.

Hence, we can say that the comparative statics effects of increasing risk aversion and imprecision aversion on the performance of flexible versus rigid contract go in the same direction, even though we remarked after Proposition 3 that the optimal flexible contracts do differ under these two specifications.

\section{Concluding remarks}

Starting from Dow and Werlang (1992) a strand of literature has shown how ambiguity (or imprecision) and ambiguity aversion can deliver qualitatively different predictions from models embedding risk and risk aversion only. The present paper contributes to this literature in the framework of a contracting problem, ${ }^{23}$ where a principal considers delegating some tasks to an agent in a situation where the environment may appear as relatively 'new' or uncertain to both parties. The possible benefits of delegation stem from the fact that the agent has some superior information, as he receives a private signal regarding the relative profitability of the various actions which can be undertaken, but the agent's action choice is not observable by the principal.

We established that imprecision aversion leads, under certain conditions, to the following properties of a flexible (delegation) contract: the compensation paid to the agent is such that the agent is fully insured against different realizations of the signal he receives as well as against different levels of the output resulting from the agent's action in some states. In contrast, this is never the case under risk aversion, where variability across signal realizations as well as across output realizations is the rule. We also showed that the higher the agent's risk aversion is, the more expensive the use of a flexible contract proves for the principal. Hence we shall observe rigid contracts being adopted more often in this case as the latter are not affected by risk aversion. An analogous result obtains under imprecision aversion, in spite of the different features of the flexible contracts in

\footnotetext{
${ }^{23}$ See e.g. for previous studies, Mukerji (1998) and Mukerji and Tallon (2004)
} 
the two cases, described above, and the fact that the agent is fully insured against various realizations of the uncertainty: when imprecision aversion is higher, the profits of a principal adopting a flexible contract are lower and hence the use of a rigid contract may become preferable. 


\section{References}

[1] Aghion, P. and J. Tirole (1997): Formal and Real Authority in Organizations, Journal of Political Economy 105, 1-29.

[2] Ahn, D., S. Choi, D. Gale and S. Kariv (2014): Estimating ambiguity aversion in a portfolio choice experiment, Quantitative Economics, 5, 195-223.

[3] Armstrong, M. and J. Vickers (2009): A Model of Delegated Project Choice, mimeo.

[4] Alonso R. and N. Matouschek (2008): Optimal Delegation, Review of Economic Studies, 75(1), 259-294.

[5] Baker, G. and B. Jorgensen (2003): Volatility, Noise and Incentives, mimeo.

[6] Billot, A., A. Chateauneuf, I. Gilboa and J-M. Tallon (2000): Sharing Beliefs: Between Agreeing and Disagreeing, Econometrica, 68(4), 685-694.

[7] Dessein, W. (2002): Authority and Communication in Organizations, Review of Economic Studies 69, 811-838.

[8] Eliaz, K. and R. Spiegler (2007): A Mechanism Design Approach to Speculative Trade, Econometrica 75(3), 875-884.

[9] Gajdos, T., T. Hayashi, J.-M. Tallon and J.-C. Vergnaud (2008): Attitude toward Imprecise Information, Journal of Economic Theory 140(1), 23-56.

[10] Ghirardato, P. (1994): Agency Theory with Non-Additive Uncertainty, mimeo, http://web.econ.unito.it/gma/paolo/age.pdf

[11] Ghirardato, P., F. Maccheroni and M. Marinacci. (2004): Differentiating Ambiguity and Ambiguity Attitude, Journal of Economic Theory 118, 133-173.

[12] Gilboa, I., and D. Schmeidler (1989): Maxmin Expected Utility with a Non-Unique Prior, Journal of Mathematical Economics 18, 141-153.

[13] Hart, O. and J. Moore (2008): Contracts as Reference Points, Quarterly Journal of Economics 123(1), 1-48. 
[14] Holmstrom, B. (1984): On the Theory of Delegation, in Bayesian Models in Economic Theory, ed. by M. Boyer and R. Kihlstrom. Elsevier, Amsterdam.

[15] Jaffray, J-Y. (1989): Linear Utility Theory for Belief Functions, Operations Research Letters, 8, 107-112.

[16] Jensen, M. and W. Meckling (1992): Specific and General Knwoledge and Organizational Structure, in Contract Economics, L. Werin and H. Wijkander eds., Oxford, Blackwell.

[17] Jullien, B., B. Salanié and F. Salanié (1999): Should More Risk-Averse Agents Exert More Effort?, The Geneva Papers on Risk and Insurance Theory 24, 19-28.

[18] Mukerji, S. (1998): Ambiguity Aversion and Incompleteness of Contractual Form, American Economic Review 88(5), 1207-3.

[19] Mukerji, S. and J.-M. Tallon (1998): Ambiguity Aversion and the Absence of Wage Indexation, Journal of Monetary Economics 51(3), 653-670.

[20] Prendergast, C. (2002): The Tenuous Trade-Off between Risk and Incentives, Journal of Political Economy 110(5), 1071-1102.

[21] Raith, M. (2008): Specific Knowledge and Performance Measurement, Rand Journal of Economics, 1059-1079.

[22] Rantakari, H.V: (2008): On the Role of Uncertainty in the Risk-Incentives Trade-off, B.E. Journal of Theoretical Economics (Topics), 8 (1), Article 10.

[23] Strzalecki, T. and J. Werner (2011): Efficient Allocations under Ambiguity, Journal of Economic Theory, 2011.

[24] Szalay, D. (2005): The Economics of Extreme Options and Clear Advice, Review of Economic Studies 72, 1173-1198. 


\section{Appendix}

\section{Proof of Proposition 1}

The proof is decomposed into three Propositions (A.1 to A.3)

Proposition A.1: At an optimal flexible contract the compensation exhibits the following properties: $\bar{w}_{1} \geq \bar{w}_{2} \geq \underline{w}_{2} \geq \underline{w}_{1}$, and $\bar{w}_{1}>\underline{w}_{1}$. Furthermore:

(i) if $\underline{w}_{2}>\underline{w}_{1}$, then $\bar{w}_{1}>\bar{w}_{2}$ and (IC3) and (IC4) are binding, while (IC1), (IC2), (IC5) and (IC6) are slack.

(ii) if $\underline{w}_{2}=\underline{w}_{1}$, then $\bar{w}_{1}=\bar{w}_{2}$ and (IC3) binds, while (IC1), (IC2), and (IC4) are automatically satisfied ((IC1) and (IC4) as equalities), and (IC5) and (IC6) are slack. $^{24}$

\section{Proof.}

Step 1: At an optimal solution $\bar{w}_{2} \geq \underline{w}_{2}$.

Proof. Suppose not, that is, $\bar{w}_{2}<\underline{w}_{2}$.

Then, it is immediate to show, given that $c_{y}<c_{x}, \pi\left(y, \theta_{1}\right)<\pi\left(x, \theta_{1}\right)$, and $\pi\left(y, \theta_{2}\right)<$ $\pi\left(x, \theta_{2}\right)$, that both (IC1) and (IC5) are slack. Start with (IC1): the right hand side of (IC1) is strictly greater than the right hand side of (IC3) and hence, (IC1) is slack. For (IC5), rewrite the constraint as:

$$
\left[\pi\left(y, \theta_{2}\right) e^{-a \bar{w}_{2}}+\left(1-\pi\left(y, \theta_{2}\right)\right) e^{-a \underline{w}_{2}}\right] e^{a c_{y}} \leq\left[\pi\left(x, \theta_{2}\right) e^{-a \bar{w}_{2}}+\left(1-\pi\left(x, \theta_{2}\right)\right) e^{-a \underline{w}_{2}}\right] e^{a c_{x}}
$$

Then, under the assumption, the expression in bracket in the left hand side is strictly smaller than the one in the right hand side, which implies, together with the order on the cost, that (IC5) is slack.

We now show that if $\bar{w}_{2}<\underline{w}_{2}$, then it is possible to find an improvement for the principal by pushing $\bar{w}_{2}$ and $\underline{w}_{2}$ closer. Consider $\Delta \bar{w}_{2}>0$ and $\Delta \underline{w}_{2}<0$ (i.e. a discrete change in $\left.\bar{w}_{2}, \underline{w}_{2}\right)$ such that:

(i) $\pi\left(y, \theta_{2}\right) e^{-a\left(\bar{w}_{2}+\Delta \bar{w}_{2}-c_{y}\right)}+\left(1-\pi\left(y, \theta_{2}\right)\right) e^{-a\left(\underline{w}_{2}+\Delta \underline{w}_{2}-c_{y}\right)}=\pi\left(y, \theta_{2}\right) e^{-a\left(\bar{w}_{2}-c_{y}\right)}+(1-$ $\left.\pi\left(y, \theta_{2}\right)\right) e^{-a\left(\underline{w}_{2}-c_{y}\right)}$ and

(ii) $\pi\left(y, \theta_{2}\right) \Delta \bar{w}_{2}+\left(1-\pi\left(y, \theta_{2}\right)\right) \Delta \underline{w}_{2}<0$

\footnotetext{
${ }^{24}$ The argument shows that the stated result holds whenever the agent's utility function can be decomposed as $u(w-c)=u(w) u(-c)$ with $u$ (strictly) concave and increasing (i.e. not only for , CARA).
} 
Note that it is possible to find such a $\Delta \bar{w}_{2}$ and $\Delta \underline{w}_{2}$ by concavity of the utility function. By condition (ii), we can conclude that this change improves the principal's profit. It remains to show that it is feasible and satisfies the remaining incentive and the participation constraints.

(IC2) is trivially satisfied since it does not depend on $\Delta \bar{w}_{2}$ and $\Delta \underline{w}_{2}$. (IC4) and (IC6) are satisfied by construction, given condition (i) and the same is true for (PC). Thus, it remains to show that (IC3) holds. Given that the left hand side of (IC3) remains unchanged, it is enough to show that:

$\pi\left(y, \theta_{1}\right) e^{-a\left(\bar{w}_{2}-c_{y}\right)}+\left(1-\pi\left(y, \theta_{1}\right)\right) e^{-a\left(\underline{w}_{2}-c_{y}\right)} \leq \pi\left(y, \theta_{1}\right) e^{-a\left(\bar{w}_{2}+\Delta \bar{w}_{2}-c_{y}\right)}+\left(1-\pi\left(y, \theta_{1}\right)\right) e^{-a\left(\underline{w}_{2}+\Delta \underline{w}_{2}-c_{y}\right)}$

This follows from condition (i) and the fact that $\pi\left(y, \theta_{1}\right)<\pi\left(y, \theta_{2}\right)$. Indeed, (i) is equivalent to $\pi\left(y, \theta_{2}\right)\left[e^{-a\left(\bar{w}_{2}+\Delta \bar{w}_{2}\right)}-e^{-a \bar{w}_{2}}\right]+\left(1-\pi\left(y, \theta_{2}\right)\right)\left[e^{-a\left(\underline{w}_{2}+\Delta \underline{w}_{2}\right)}-e^{-a \underline{w}_{2}}\right]=0$. The first term is negative while the second is positive, so we have, given that $\pi\left(y, \theta_{1}\right)<\pi\left(y, \theta_{2}\right)$,

$\pi\left(y, \theta_{1}\right)\left[e^{-a\left(\bar{w}_{2}+\Delta \bar{w}_{2}\right)}-e^{-a \bar{w}_{2}}\right]+\left(1-\pi\left(y, \theta_{1}\right)\right)\left[e^{-a\left(\underline{w}_{2}+\Delta \underline{w}_{2}\right)}-e^{-a \underline{w}_{2}}\right]>0$, which yields the desired result.

Step 2: At an optimal solution $\bar{w}_{1}>\underline{w}_{1}$.

Proof. This is a direct consequence of (IC2).

Step 3: At an optimal solution (IC3) binds.

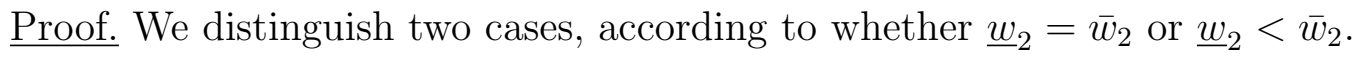

Case $1 .: \underline{w}_{2}=\bar{w}_{2} \equiv w_{2}$.

In that event, (IC5) is automatically satisfied and therefore can be dropped. Furthermore, (IC3) implies (IC1) which can so also be dropped. Now, by Step $2 \underline{w}_{1}<\bar{w}_{1}$. Hence, given that $\pi\left(y, \theta_{2}\right)>\pi\left(y, \theta_{1}\right)$, it is possible to show that (IC3) and (IC4) imply (IC2), which can be dropped.

Obviously, (IC3) and (IC6) cannot be simultaneously binding. We show next that (IC3) has to bind and therefore (IC6) is slack. Assume not, i.e., (IC3) is slack and consider (an infinitesimal change) $d \bar{w}_{1}<0, d \underline{w}_{1}=0$ and $d w_{2}>0$. Since (IC3) is slack, for sufficiently small such quantities it continues to hold. (IC4) and (IC6) remain satisfied. Choosing $d \bar{w}_{1}=-\frac{(1-p) e^{-a\left(w_{2}-c_{y}\right)}}{p \pi\left(x, \theta_{1}\right) e^{-a\left(\bar{w}_{1}-c_{x}\right)}} d w_{2}$ ensures that the participation constraint continues to hold. By construction, the change in the objective function is equal to $(1-p)\left[\frac{e^{-a\left(w_{2}-c_{y}\right)}}{e^{-a\left(\bar{w}_{1}-c_{x}\right)}}-1\right] d w_{2}$. Given that $d w_{2}>0$, this quantity is positive (hence leading to an increase in the objective function) if $e^{-a\left(w_{2}-c_{y}\right)}>e^{-a\left(\bar{w}_{1}-c_{x}\right)}$, that is if $\bar{w}_{1}>w_{2}+\Delta c$. 
This property always holds in the case under consideration $\left(\underline{w}_{2}=\bar{w}_{2}\right)$ : (IC3) can in fact be rewritten as follows:

$$
\pi\left(x, \theta_{1}\right) e^{-a \bar{w}_{1}}+\left(1-\pi\left(x, \theta_{1}\right)\right) e^{-a \underline{w}_{1}} \leq e^{-a\left(w_{2}+\Delta c\right)},
$$

which in turn implies, together with the property $\bar{w}_{1}>\underline{w}_{1}$ established in Step 2, that $e^{-a \bar{w}_{1}}<e^{-a\left(w_{2}+\Delta c\right)}$, and therefore $\bar{w}_{1}>w_{2}+\Delta c$.

Hence, whenever (IC3) is slack we can find a perturbation of the wage bill that increases the principal's profit, contradicting optimality of the contract. Therefore (IC3) has to bind (and hence (IC6) is slack).

Case $2 .: \underline{w}_{2}<\bar{w}_{2}$.

Assume (IC3) is slack and consider a discrete change $\Delta \underline{w}_{2}>0$ and $\Delta \bar{w}_{2}<0$ such that: (i) $\pi\left(y, \theta_{2}\right) \Delta \bar{w}_{2}+\left(1-\pi\left(y, \theta_{2}\right) \Delta \underline{w}_{2}<0\right.$ and (ii) $\pi\left(y, \theta_{2}\right) e^{-a\left(\bar{w}_{2}+\Delta \bar{w}_{2}\right)}+\left(1-\pi\left(y, \theta_{2}\right)\right) e^{-a\left(\bar{w}_{2}+\Delta \underline{w}_{2}\right)}=$ $\pi\left(y, \theta_{2}\right) e^{-a \bar{w}_{2}}+\left(1-\pi\left(y, \theta_{2}\right)\right) e^{-a \bar{w}_{2}}$. Such numbers exist by strict concavity of $u$.

Notice that (IC2), (IC4), (IC6) and (PC) are unaffected by these changes and thus continue to hold. We now check (IC1). The left hand side is unchanged and we therefore need to show that: $\pi\left(x, \theta_{1}\right) e^{-a\left(\bar{w}_{2}-c_{x}\right)}+\left(1-\pi\left(x, \theta_{1}\right)\right) e^{-a\left(\underline{w}_{2}-c_{x}\right)} \leq \pi\left(x, \theta_{1}\right) e^{-a\left(\bar{w}_{2}+\Delta \bar{w}_{2}-c_{x}\right)}+$ $\left(1-\pi\left(x, \theta_{1}\right)\right) e^{-a\left(\underline{w}_{2}+\Delta \underline{w}_{2}-c_{x}\right)}$, which is equivalent to

$$
\pi\left(x, \theta_{1}\right)\left[e^{-a \bar{w}_{2}}-e^{-a\left(\bar{w}_{2}+\Delta \bar{w}_{2}\right)}\right]+\left(1-\pi\left(x, \theta_{1}\right)\right)\left[e^{-a \underline{w}_{2}}-e^{-a\left(\underline{w}_{2}+\Delta \underline{w}_{2}\right)}\right] \leq 0
$$

But this holds as a consequence of (ii), given that $\Delta \underline{w}_{2}>0$ and $\Delta \bar{w}_{2}<0$ and $\pi\left(x, \theta_{1}\right)>$ $\pi\left(y, \theta_{2}\right)$. Thus, (IC1) continues to hold.

It remains to check (IC5). By construction, the left hand side is unaffected by the change. Given that $\pi\left(x, \theta_{2}\right)>\pi\left(y, \theta_{2}\right)$, one can replicate the argument showing that (IC1) holds to prove that (IC5) holds as well.

Step 4: At an optimal solution (IC6) is slack.

Proof. Given that $\bar{w}_{2} \geq \underline{w}_{2}$ and $\pi\left(y, \theta_{2}\right) \geq \pi\left(y, \theta_{1}\right)$, we have

$$
\pi\left(y, \theta_{2}\right) e^{-a\left(\bar{w}_{2}-c_{y}\right)}+\left(1-\pi\left(y, \theta_{2}\right)\right) e^{-a\left(\underline{w}_{2}-c_{y}\right)} \leq \pi\left(y, \theta_{1}\right) e^{-a\left(\bar{w}_{2}-c_{y}\right)}+\left(1-\pi\left(y, \theta_{1}\right)\right) e^{-a\left(\underline{w}_{2}-c_{y}\right)} .
$$

From the previous step, we know (IC3) is binding, and hence

$$
\pi\left(y, \theta_{2}\right) e^{-a\left(\bar{w}_{2}-c_{y}\right)}+\left(1-\pi\left(y, \theta_{2}\right)\right) e^{-a\left(\underline{w}_{2}-c_{y}\right)} \leq \pi\left(x, \theta_{1}\right) e^{-a\left(\bar{w}_{1}-c_{x}\right)}+\left(1-\pi\left(x, \theta_{1}\right)\right) e^{-a\left(\underline{w}_{1}-c_{x}\right)}
$$

Given that $\bar{w}_{1}>\underline{w}_{1}$ and $\pi\left(x, \theta_{1}\right) \geq \pi\left(x, \theta_{2}\right)$, this establishes that (IC6) is slack, i.e.

$$
\pi\left(y, \theta_{2}\right) e^{-a\left(\bar{w}_{2}-c_{y}\right)}+\left(1-\pi\left(y, \theta_{2}\right)\right) e^{-a\left(\underline{w}_{2}-c_{y}\right)}<\pi\left(x, \theta_{2}\right) e^{-a\left(\bar{w}_{1}-c_{x}\right)}+\left(1-\pi\left(x, \theta_{2}\right)\right) e^{-a\left(\underline{w}_{1}-c_{x}\right)}
$$


Step 5: At an optimal solution (IC5) is slack.

Proof. If $\bar{w}_{2}=\underline{w}_{2}$, this is obvious. Consider next the case $\bar{w}_{2}>\underline{w}_{2}$. Then, $\pi\left(y, \theta_{2}\right) e^{-a\left(\bar{w}_{2}-c_{y}\right)}+$ $\left(1-\pi\left(y, \theta_{2}\right)\right) e^{-a\left(\underline{w}_{2}-c_{y}\right)} \leq \pi\left(y, \theta_{1}\right) e^{-a\left(\bar{w}_{2}-c_{y}\right)}+\left(1-\pi\left(y, \theta_{1}\right)\right) e^{-a\left(\underline{w}_{2}-c_{y}\right)}$. From Step 3 we know that (IC2) binds, i.e., $\pi\left(y, \theta_{1}\right) e^{-a\left(\bar{w}_{2}-c_{y}\right)}+\left(1-\pi\left(y, \theta_{1}\right)\right) e^{-a\left(\underline{w}_{2}-c_{y}\right)}=\pi\left(x, \theta_{1}\right) e^{-a\left(\bar{w}_{1}-c_{x}\right)}+$ $\left(1-\pi\left(x, \theta_{1}\right)\right) e^{-a\left(\underline{w}_{1}-c_{x}\right)}$.

Now, by (IC1), $\pi\left(x, \theta_{1}\right) e^{-a\left(\bar{w}_{1}-c_{x}\right)}+\left(1-\pi\left(x, \theta_{1}\right)\right) e^{-a\left(\underline{w}_{1}-c_{x}\right)} \leq \pi\left(x, \theta_{1}\right) e^{-a\left(\bar{w}_{2}-c_{x}\right)}+(1-$ $\left.\pi\left(x, \theta_{1}\right)\right) e^{-a\left(\underline{w}_{2}-c_{x}\right)}$ and hence, since $\bar{w}_{2}>\underline{w}_{2}$ and $\pi\left(x, \theta_{1}\right)>\pi\left(x, \theta_{2}\right), \pi\left(x, \theta_{1}\right) e^{-a\left(\bar{w}_{1}-c_{x}\right)}+$ $\left(1-\pi\left(x, \theta_{1}\right)\right) e^{-a\left(\underline{w}_{1}-c_{x}\right)}<\pi\left(x, \theta_{2}\right) e^{-a\left(\bar{w}_{2}-c_{x}\right)}+\left(1-\pi\left(x, \theta_{2}\right)\right) e^{-a\left(\underline{w}_{2}-c_{x}\right)}$. As a consequence, $\pi\left(y, \theta_{2}\right) e^{-a\left(\bar{w}_{2}-c_{y}\right)}+\left(1-\pi\left(y, \theta_{2}\right)\right) e^{-a\left(\underline{w}_{2}-c_{y}\right)}<\pi\left(x, \theta_{2}\right) e^{-a\left(\bar{w}_{2}-c_{x}\right)}+\left(1-\pi\left(x, \theta_{2}\right)\right) e^{-a\left(\underline{w}_{2}-c_{x}\right)}$ showing that (IC5) is slack.

Step 6: At an optimal solution, $\bar{w}_{1} \geq \bar{w}_{2}$ and $\underline{w}_{1} \leq \underline{w}_{2}$. Furthermore, if $\underline{w}_{1}=\underline{w}_{2}$, then it must be the case that $\bar{w}_{1}=\bar{w}_{2}$.

Proof. Rewrite (IC1) and (IC4) as follows:

$$
\begin{aligned}
& \pi\left(x, \theta_{1}\right)\left[e^{-a \bar{w}_{1}}-e^{-a \bar{w}_{2}}\right] \leq\left(1-\pi\left(x, \theta_{1}\right)\right)\left[e^{-a \underline{w}_{2}}-e^{-a \underline{w}_{1}}\right] \\
& \pi\left(y, \theta_{2}\right)\left[e^{-a \bar{w}_{2}}-e^{-a \bar{w}_{1}}\right] \leq\left(1-\pi\left(y, \theta_{2}\right)\right)\left[e^{-a \underline{w}_{1}}-e^{-a \underline{w}_{2}}\right]
\end{aligned}
$$

Assume $\bar{w}_{1}<\bar{w}_{2}$, then (6) implies that $\underline{w}_{1}>\underline{w}_{2}$ and (6) and (7) yield that:

$$
\frac{\pi\left(x, \theta_{1}\right)}{1-\pi\left(x, \theta_{1}\right)} \leq \frac{e^{-a \underline{w}_{2}}-e^{-a \underline{w}_{1}}}{e^{-a \bar{w}_{1}}-e^{-a \bar{w}_{2}}} \leq \frac{\pi\left(y, \theta_{2}\right)}{1-\pi\left(y, \theta_{2}\right)}
$$

But this is not possible given that $\pi\left(y, \theta_{2}\right)<\pi\left(x, \theta_{1}\right)$. Hence, $\bar{w}_{1} \geq \bar{w}_{2}$. A similar argument establishes that $\underline{w}_{1} \leq \underline{w}_{2}$.

Finally, suppose that $\underline{w}_{1}=\underline{w}_{2}$. Then, using the fact that (IC3) is binding, one can rewrite (IC2) as follows:

$$
\pi\left(y, \theta_{1}\right) e^{-a\left(\bar{w}_{2}-c_{y}\right)}+\left(1-\pi\left(y, \theta_{1}\right)\right) e^{-a\left(\underline{w}_{2}-c_{y}\right)} \leq \pi\left(y, \theta_{1}\right) e^{-a\left(\bar{w}_{1}-c_{y}\right)}+\left(1-\pi\left(y, \theta_{1}\right)\right) e^{-a\left(\underline{w}_{1}-c_{y}\right)}
$$

which yields $\bar{w}_{1}=\bar{w}_{2}$, since we assumed that $\underline{w}_{1}=\underline{w}_{2}$ and we proved above that $\bar{w}_{1} \geq$ $\bar{w}_{2}$.

Step 7: At an optimal solution (IC2) is slack if $\underline{w}_{1}<\underline{w}_{2}$. If $\underline{w}_{1}=\underline{w}_{2}$, (IC2) is automatically satisfied as equality. 
Proof. Use (IC3), which is binding, to rewrite (IC2) as follows:

$$
\pi\left(y, \theta_{1}\right) e^{-a\left(\bar{w}_{2}-c_{y}\right)}+\left(1-\pi\left(y, \theta_{1}\right)\right) e^{-a\left(\underline{w}_{2}-c_{y}\right)} \leq \pi\left(y, \theta_{1}\right) e^{-a\left(\bar{w}_{1}-c_{y}\right)}+\left(1-\pi\left(y, \theta_{1}\right)\right) e^{-a\left(\underline{w}_{1}-c_{y}\right)}
$$

If $\underline{w}_{1}<\underline{w}_{2}$, (8) is equivalent, given that $\bar{w}_{1} \geq \bar{w}_{2}$, to

$$
\frac{e^{-a \bar{w}_{2}}-e^{-a \bar{w}_{1}}}{e^{-a \underline{w}_{1}}-e^{-a \underline{w}_{2}}} \leq \frac{1-\pi\left(y, \theta_{1}\right)}{\pi\left(y, \theta_{1}\right)}
$$

But we know by (IC4) that

$$
\frac{e^{-a \bar{w}_{2}}-e^{-a \bar{w}_{1}}}{e^{-a \underline{w}_{1}}-e^{-a \underline{w}_{2}}} \leq \frac{1-\pi\left(y, \theta_{2}\right)}{\pi\left(y, \theta_{2}\right)}
$$

and hence, since $\pi\left(y, \theta_{1}\right)<\pi\left(y, \theta_{2}\right)$, (IC2) is slack.

If $\underline{w}_{1}=\underline{w}_{2}$, then we know that $\bar{w}_{1}=\bar{w}_{2}$ and (8) - hence (IC2) - is automatically satisfied.

Step 8: At an optimal solution (IC1) and (IC4) cannot be simultaneously binding if $\underline{w}_{1}<\underline{w}_{2}$. If $\underline{w}_{1}=\underline{w}_{2}$ they are both automatically satisfied (as equalities).

Proof. Assume $\underline{w}_{1}<\underline{w}_{2}$ and observe that if (IC1) and (IC4) were binding, one would have

$$
\frac{1-\pi\left(x, \theta_{1}\right)}{\pi\left(x, \theta_{1}\right)}=\frac{e^{-a \bar{w}_{2}}-e^{-a \bar{w}_{1}}}{e^{-a \underline{w}_{1}}-e^{-a \underline{w}_{2}}}=\frac{1-\pi\left(y, \theta_{2}\right)}{\pi\left(y, \theta_{2}\right)}
$$

a contradiction.

Step 9: At an optimal solution, if $\underline{w}_{1}<\underline{w}_{2}$ (IC4) binds.

Proof. Assume $\underline{w}_{1}<\underline{w}_{2}$ and (IC4) is slack and consider changing $\bar{w}_{1}$ and $\underline{w}_{1}$ by respectively $\Delta \bar{w}_{1}<0$ and $\Delta \underline{w}_{1}>0$ such that, (i) $\pi\left(x, \theta_{1}\right) \Delta \bar{w}_{1}+\left(1-\pi\left(x, \theta_{1}\right)\right) \Delta \underline{w}_{1}<0$ and (ii), $\pi\left(x, \theta_{1}\right) e^{-a\left(\bar{w}_{1}+\Delta \bar{w}_{1}\right)}+\left(1-\pi\left(x, \theta_{1}\right)\right) e^{-a\left(\underline{w}_{1}+\Delta \underline{w}_{1}\right)}=\pi\left(x, \theta_{1}\right) e^{-a \bar{w}_{1}}+\left(1-\pi\left(x, \theta_{1}\right)\right) e^{-a \underline{w}_{1}}$. Such a change exists by strict concavity of the utility function and provides higher profit to the principal.

Furthermore, this change does not affect (IC1), (IC3), and (PC) and is feasible given that (IC2), (IC4), (IC5) and (IC6) are slack. Hence, (IC4) has to be binding at an optimal solution whenever $\underline{w}_{1}<\underline{w}_{2}$.

Steps 1-9 complete the proof of Proposition A.1. From this result it then immediately follows: 
Corollary A.1: The optimal flexible contract can be obtained as a solution to the simpler programme below:

$$
\begin{aligned}
& \begin{aligned}
\left.\max _{\bar{w}_{1}, \underline{w}_{1}, \bar{w}_{2}, \underline{w}_{2} \quad p[} \quad \pi\left(x, \theta_{1}\right)\left(\bar{R}-\bar{w}_{1}\right)+\left(1-\pi\left(x, \theta_{1}\right)\right)\left(\underline{R}-\underline{w}_{1}\right)\right] \\
+(1-p)\left[\pi\left(y, \theta_{2}\right)\left(\bar{R}-\bar{w}_{2}\right)+\left(1-\pi\left(y, \theta_{2}\right)\right)\left(\underline{R}-\underline{w}_{2}\right)\right]
\end{aligned} \\
& \text { s.t. } \\
& \left\{\begin{array}{l}
\left.(I C 3),(I C 4),(P C) \text { (as stated in }\left(P^{\text {flex }}\right)\right) \text { and } \\
(W I) \quad \bar{w}_{1} \geq \bar{w}_{2} \\
(W I I) \quad \bar{w}_{2} \geq \underline{w}_{2}
\end{array}\right. \\
& \left(P^{f l e x, R}\right)
\end{aligned}
$$

Observe the constraint $\underline{w}_{2} \geq \underline{w}_{1}$ is implied by $(W I)$ and $(I C 4)$.

Proposition A.2: $\quad$ Under Assumption 1, 2, there exists a solution to problem $\left(P^{\text {flex,R}}\right)$ (and hence also to $\left.\left(P^{\text {flex }}\right)\right)$.

Proof. The two binding constraints (IC3) and (IC4) enable one to solve for $\bar{z}_{1}=e^{-a \bar{w}_{1}}$ and $\underline{z}_{1} \equiv e^{-a \underline{w}_{1}}$ as a function of $\bar{z}_{2} \equiv e^{-a \bar{w}_{2}}$ and $\underline{z}_{2} \equiv e^{-a \underline{w}_{2}}$, yielding:

$$
\begin{aligned}
& \bar{z}_{1}=\frac{\left(\left(1-\pi\left(y, \theta_{2}\right)\right)\left[\pi\left(y, \theta_{1}\right) \bar{z}_{2}+\left(1-\pi\left(y, \theta_{1}\right)\right) \underline{z}_{2}\right] e^{-a \Delta c}-\left(1-\pi\left(x, \theta_{1}\right)\right)\left[\pi\left(y, \theta_{2}\right) \bar{z}_{2}+\left(1-\pi\left(y, \theta_{2}\right)\right) \underline{z}_{2}\right]\right)}{\pi\left(x, \theta_{1}\right)-\pi\left(y, \theta_{2}\right)} \\
& \underline{z}_{1}=\frac{\left(\pi\left(x \theta_{1}\right)\left[\pi\left(y, \theta_{2}\right) \bar{z}_{2}+\left(1-\pi\left(y, \theta_{2}\right)\right) \underline{z}_{2}\right]-\pi\left(y, \theta_{2}\right)\left[\pi\left(y, \theta_{1}\right) \bar{z}_{2}+\left(1-\pi\left(y, \theta_{1}\right)\right) \underline{z}_{2}\right] e^{-a \Delta c}\right)}{\pi\left(x, \theta_{1}\right)-\pi\left(y, \theta_{2}\right)}
\end{aligned}
$$

We now want to establish that under the condition $\frac{1-\pi\left(y, \theta_{1}\right)}{1-\pi\left(x, \theta_{1}\right)} \geq e^{a \Delta c}$, it is possible to find $0 \leq \bar{z}_{2} \leq \underline{z}_{2}$ such that:

$$
\begin{aligned}
& \bar{z}_{1}>0 \\
& \bar{z}_{1} \leq \bar{z}_{2} \\
& \underline{z}_{2} \leq \underline{z}_{1} \\
& \bar{z}_{2} \leq \underline{z}_{2}
\end{aligned}
$$

These inequalities ensure that values of the wages satisfying $\bar{w}_{1} \geq \bar{w}_{2} \geq \underline{w}_{2} \geq \underline{w}_{1}$ can be found.

The first inequality is equivalent, under the condition $\frac{1-\pi\left(y, \theta_{1}\right)}{1-\pi\left(x, \theta_{1}\right)} \geq e^{a \Delta c}$, to

$$
\frac{\left(1-\pi\left(x, \theta_{1}\right)\right) \pi\left(y, \theta_{2}\right)-\left(1-\pi\left(y, \theta_{2}\right)\right) \pi\left(y, \theta_{1}\right) e^{-a \Delta c}}{\left(1-\pi\left(y, \theta_{2}\right)\right)\left[\left(1-\pi\left(y, \theta_{1}\right)\right) e^{-a \Delta c}-\left(1-\pi\left(x, \theta_{1}\right)\right)\right]}<\frac{\underline{z}_{2}}{\bar{z}_{2}}
$$


The next two inequalities are actually equivalent (again under the condition $\frac{1-\pi\left(y, \theta_{1}\right)}{1-\pi\left(x, \theta_{1}\right)} \geq$ $\left.e^{a \Delta c}\right)$ to the same inequality:

$$
\frac{\pi\left(x, \theta_{1}\right)-\pi\left(y, \theta_{1}\right) e^{-a \Delta c}}{\left(1-\pi\left(y, \theta_{1}\right)\right) e^{-a \Delta c}-\left(1-\pi\left(x, \theta_{1}\right)\right)} \geq \frac{\underline{z}_{2}}{\bar{z}_{2}}
$$

Thus, to show that we can find some values $\bar{z}_{2}, \underline{z}_{2}$ satisfying the last inequality, $\bar{z}_{2} \leq \underline{z}_{2}$, and such that (9) and (10) hold, we need to establish that the following holds:

$\max \left(1, \frac{\left(1-\pi\left(x, \theta_{1}\right)\right) \pi\left(y, \theta_{2}\right)-\left(1-\pi\left(y, \theta_{2}\right)\right) \pi\left(y, \theta_{1}\right) e^{-a \Delta c}}{\left(1-\pi\left(y, \theta_{2}\right)\right)\left[\left(1-\pi\left(y, \theta_{1}\right)\right) e^{-a \Delta c}-\left(1-\pi\left(x, \theta_{1}\right)\right)\right]}\right)<\frac{\pi\left(x, \theta_{1}\right)-\pi\left(y, \theta_{1}\right) e^{-a \Delta c}}{\left(1-\pi\left(y, \theta_{1}\right)\right) e^{-a \Delta c}-\left(1-\pi\left(x, \theta_{1}\right)\right)}$

Straightforward computation shows that, under the assumption that $\frac{1-\pi\left(y, \theta_{1}\right)}{1-\pi\left(x, \theta_{1}\right)} \geq e^{a \Delta c}$, this is indeed the case.

Before solving problem $\left(P^{f l e x, R}\right)$, observe that one can rewrite it, with the following change of variables $z=e^{-a w}$, as a problem with a (strictly) concave objective and linear constraints:

$$
\begin{aligned}
& \max _{\bar{z}_{1}, \underline{z}_{1}, \bar{z}_{2}, \underline{z}_{2}} \quad p\left[\pi\left(x, \theta_{1}\right)\left(\bar{R}+\frac{\log \bar{z}_{1}}{a}\right)+\left(1-\pi\left(x, \theta_{1}\right)\right)\left(\underline{R}+\frac{\log \underline{z}_{1}}{a}\right)\right] \\
& +(1-p)\left[\pi\left(y, \theta_{2}\right)\left(\bar{R}+\frac{\log \bar{z}_{2}}{a}\right)+\left(1-\pi\left(y, \theta_{2}\right)\right)\left(\underline{R}+\frac{\log \underline{z}_{2}}{a}\right)\right] \\
& \left\{\begin{array}{cc}
\left(I C 3^{\prime}\right) & \pi\left(x, \theta_{1}\right) e^{a c_{x}} \bar{z}_{1}+\left(1-\pi\left(x, \theta_{1}\right)\right) e^{a c_{x}} \underline{z}_{1}=\pi\left(y, \theta_{1}\right) e^{a c_{y}} \bar{z}_{2}+\left(1-\pi\left(y, \theta_{1}\right)\right) e^{a c_{y}} \underline{z}_{2} \\
\left(I C 4^{\prime}\right) & \pi\left(y, \theta_{2}\right) e^{a c_{y}} \bar{z}_{2}+\left(1-\pi\left(y, \theta_{2}\right)\right) e^{a c_{y}} \underline{z}_{2}=\pi\left(y, \theta_{2}\right) e^{a c_{y}} \bar{z}_{1}+\left(1-\pi\left(y, \theta_{2}\right)\right) e^{a c_{y}} \underline{z}_{1} \\
\left(P C^{\prime}\right) & p\left[\pi\left(x, \theta_{1}\right) e^{a c_{x}} \bar{z}_{1}+\left(1-\pi\left(x, \theta_{1}\right)\right) e^{a c_{x}} \underline{z}_{1}\right]+ \\
& (1-p)\left[\pi\left(y, \theta_{2}\right) e^{a c_{y}} \bar{z}_{2}+\left(1-\pi\left(y, \theta_{2}\right)\right) e^{a c_{y}} \underline{z}_{2}\right] \leq e^{-a \bar{u}}
\end{array}\right. \\
& \left(W I^{\prime}\right) \quad \bar{z}_{1} \leq \bar{z}_{2} \\
& \left(W I I^{\prime}\right) \quad \bar{z}_{2} \leq \underline{z}_{2}
\end{aligned}
$$

$$
\left(\widetilde{P}^{\text {flex }, R}\right)
$$

Proposition A.3: At a solution to the program $\left(\widetilde{P}^{\text {flex }, R}\right),\left(P C^{\prime}\right)$ binds. Furthermore, we have that $\bar{w}_{2}>\underline{w}_{2}$.

Proof. Consider the program $\left(\widetilde{P}^{f l e x, R}\right)$. Let $\lambda_{3}, \lambda_{4}, \lambda_{P C}, \lambda_{I}$, and $\lambda_{I I}$ denote the Lagrange multipliers associated to the constraints of this problem. The first order conditions obtained by differentiating the Lagrangean with respect to $\bar{z}_{1}, \bar{z}_{2}, \underline{z}_{2}, \underline{z}_{1}$ are then: 


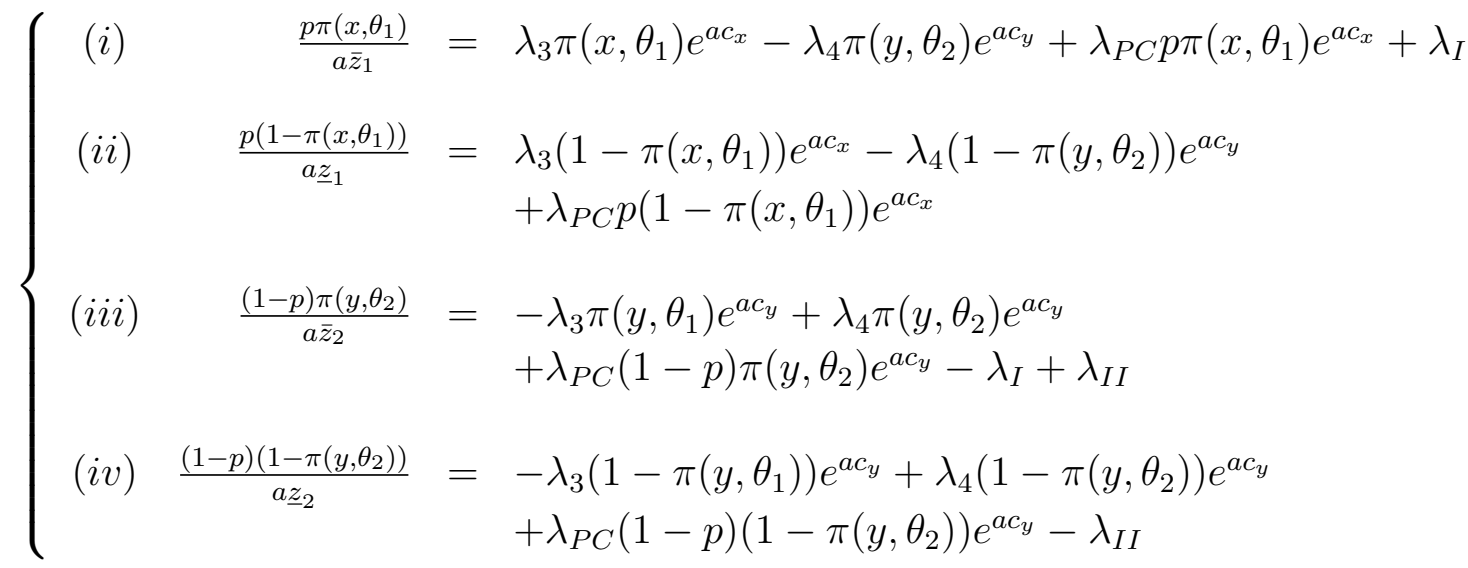

Multiplying each equation by the appropriate $z$ variable, adding the four equations of the above system and using the fact that $\left(I C 3^{\prime}\right)$ and $\left(I C 4^{\prime}\right)$, in the above specification of the optimization problem, are written as equalities, yields the following:

$$
\begin{aligned}
\underline{1}= & \lambda_{P C}\left[p \pi\left(x, \theta_{1}\right) e^{a c_{x}} \bar{z}_{1}+p\left(1-\pi\left(x, \theta_{1}\right)\right) e^{a c_{x}} \underline{z}_{1}\right. \\
& \left.+(1-p) \pi\left(y, \theta_{2}\right) e^{a c_{y}} \bar{z}_{2}+(1-p)\left(1-\pi\left(y, \theta_{2}\right)\right) e^{a c_{y}} \underline{z}_{2}\right]+\lambda_{I}\left[\bar{z}_{1}-\bar{z}_{2}\right]+\lambda_{I I}\left[\bar{z}_{2}-\underline{z}_{2}\right]
\end{aligned}
$$

Using the complementarity slackness condition, we get that $\lambda_{I}\left[\bar{z}_{1}-\bar{z}_{2}\right]=\lambda_{I I}\left[\bar{z}_{2}-\underline{z}_{2}\right]=$ 0 . Hence $\lambda_{P C}>0$, which establishes that $\left(P C^{\prime}\right)$ binds. Hence, we can conclude from the expression above that $\lambda_{P C}=\frac{e^{a \bar{u}}}{a}$.

Next we want to show that $\bar{w}_{2}>\underline{w}_{2}$ or equivalently $\underline{z}_{2}>\bar{z}_{2}$. Assume to the contrary that $\bar{z}_{2}=\underline{z}_{2} \equiv z_{2}$. We know in that case that $\left(W I^{\prime}\right)$ is slack (otherwise by $\left(I C 4^{\prime}\right)$ all wages would have to be equal, but this would contradict the fact that $\left(I C 3^{\prime}\right)$ binds) and hence $\lambda_{I}=0$. Rewrite now FOC's $(i i i)$ and $(i v)$ as:

$$
\left\{\begin{array}{l}
\text { (iii) } \frac{(1-p)}{a z_{2}}=-\lambda_{3} \frac{\pi\left(y, \theta_{1}\right)}{\pi\left(y, \theta_{2}\right)} e^{a c_{y}}+\lambda_{4} e^{a c_{y}}+\lambda_{P C}(1-p) e^{a c_{y}}+\frac{\lambda_{I I}}{\pi\left(y, \theta_{2}\right)} \\
\text { (iv) } \frac{(1-p)}{a z_{2}}=-\lambda_{3} \frac{1-\pi\left(y, \theta_{1}\right)}{1-\pi\left(y, \theta_{2}\right)} e^{a c_{y}}+\lambda_{4} e^{a c_{y}}+\lambda_{P C}(1-p) e^{a c_{y}}-\frac{\lambda_{I I}}{1-\pi\left(y, \theta_{2}\right)}
\end{array}\right.
$$

This implies that

$$
-\lambda_{3} \frac{\pi\left(y, \theta_{1}\right)}{\pi\left(y, \theta_{2}\right)} e^{a c_{y}}+\frac{\lambda_{I I}}{\pi\left(y, \theta_{2}\right)}=-\lambda_{3} \frac{1-\pi\left(y, \theta_{1}\right)}{1-\pi\left(y, \theta_{2}\right)} e^{a c_{y}}-\frac{\lambda_{I I}}{1-\pi\left(y, \theta_{2}\right)}
$$

or, after some simplification,

$$
\lambda_{I I}=\left(\pi\left(y, \theta_{1}\right)-\pi\left(y, \theta_{2}\right)\right) \lambda_{3} e^{a c_{y}}
$$

Note that $\left(\pi\left(y, \theta_{1}\right)-\pi\left(y, \theta_{2}\right)\right)<0$ and hence $\lambda_{I I} \geq 0$ iff $\lambda_{3} \leq 0$. Next observe that $\left(P C^{\prime}\right)$ as an equality together with $\left(I C 3^{\prime}\right)$ imply, if $\bar{z}_{2}=\underline{z}_{2} \equiv z_{2}$, that $z_{2}=e^{-a\left(c_{y}+\bar{u}\right)}$. 
Plug now the values of $\lambda_{P C}$ and $z_{2}$ into equations (iii) and (iv) and use the expression for $\lambda_{I I}$ obtained above. The two equations are identical and yield $\lambda_{4}=\lambda_{3} \equiv \lambda$.

We have so a system of four equations - FOC's $(i)$ and $(i i),\left(I C 3^{\prime}\right)$ and $\left(I C 4^{\prime}\right)$ - to determine three variables: $\lambda, \bar{z}_{1}$ and $\underline{z}_{1}$. $\left(I C 3^{\prime}\right)$ and $\left(I C 4^{\prime}\right)$ can be used to solve directly for $\bar{z}_{1}$ and $\underline{z}_{1}$. Now, the two FOC's can be rewritten:

$$
\begin{aligned}
p \pi\left(x, \theta_{1}\right) & =a \lambda \bar{z}_{1}\left(\pi\left(x, \theta_{1}\right) e^{a c_{x}}-\pi\left(y, \theta_{2}\right) e^{a c_{y}}\right)+e^{a \bar{u}} \bar{z}_{1} p \pi\left(x, \theta_{1}\right) e^{a c_{x}} \\
p\left(1-\pi\left(x, \theta_{1}\right)\right) & =a \lambda \underline{z}_{1}\left(\left(1-\pi\left(x, \theta_{1}\right)\right) e^{a c_{x}}-\left(1-\pi\left(y, \theta_{2}\right)\right) e^{a c_{y}}\right)+e^{a \bar{u}} \underline{z}_{1} p\left(1-\pi\left(x, \theta_{1}\right)\right) e^{a c_{x}}
\end{aligned}
$$

Adding these two equations yields an equation

$$
\begin{aligned}
p= & a \lambda\left[\bar{z}_{1}\left(\pi\left(x, \theta_{1}\right) e^{a c_{x}}+\underline{z}_{1}\left(1-\pi\left(x, \theta_{1}\right)\right) e^{a c_{x}}-\pi\left(y, \theta_{2}\right) \bar{z}_{1} e^{a c_{y}}-\underline{z}_{1}\left(1-\pi\left(y, \theta_{2}\right)\right) e^{a c_{y}}\right]+\right. \\
& +p e^{a \bar{u}}\left[\bar{z}_{1} \pi\left(x, \theta_{1}\right) e^{a c_{x}}+\underline{z}_{1}\left(1-\pi\left(x, \theta_{1}\right)\right) e^{a c_{x}}\right]
\end{aligned}
$$

which, using $\left(I C 3^{\prime}\right)$ and $\left(I C 4^{\prime}\right)$ can be rewritten as:

$$
p=a \lambda\left[e^{a c_{y}} e^{-a\left(c_{y}+\bar{u}\right)}-e^{a c_{y}} e^{-a\left(c_{y}+\bar{u}\right)}\right]+p e^{a \bar{u}}\left[e^{a c_{y}} e^{-a\left(c_{y}+\bar{u}\right)}\right]=p\left[e^{a c_{y}} e^{-a c_{y}}\right]=p
$$

always satisfied, so that one of the two above equations can be dropped. The remaining one can be used to solve for $\lambda$. Recall that $\lambda \leq 0$ is needed to ensure that $\lambda_{I I} \geq 0$.

Solving then $\left(I C 3^{\prime}\right)$ and $\left(I C 4^{\prime}\right)$ with respect to $\bar{z}_{1}$ and $\underline{z}_{1}$ we get:

$$
\begin{aligned}
& \underline{z}_{1}=\frac{\pi\left(x, \theta_{1}\right) e^{-a\left(c_{y}+\bar{u}\right)}-\pi\left(y, \theta_{2}\right) e^{-a\left(c_{x}+\bar{u}\right)}}{\pi\left(x, \theta_{1}\right)-\pi\left(y, \theta_{2}\right)} \\
& \bar{z}_{1}=\frac{\left(1-\pi\left(y, \theta_{2}\right)\right) e^{-a\left(c_{x}+\bar{u}\right)}-\left(1-\pi\left(x, \theta_{1}\right)\right) e^{-a\left(c_{y}+\bar{u}\right)}}{\pi\left(x, \theta_{1}\right)-\pi\left(y, \theta_{2}\right)} .
\end{aligned}
$$

Substituting into the first of the two FOC's above yields:

$$
\begin{aligned}
p \pi\left(x, \theta_{1}\right)= & {\left[a \lambda\left(\pi\left(x, \theta_{1}\right) e^{a c_{x}}-\pi\left(y, \theta_{2}\right) e^{a c_{y}}\right)+e^{a \bar{u}} p \pi\left(x, \theta_{1}\right) e^{a c_{x}}\right] . } \\
& \cdot \frac{\left(1-\pi\left(y, \theta_{2}\right)\right) e^{-a\left(c_{x}+\bar{u}\right)}-\left(1-\pi\left(x, \theta_{1}\right)\right) e^{-a\left(c_{y}+\bar{u}\right)}}{\pi\left(x, \theta_{1}\right)-\pi\left(y, \theta_{2}\right)}
\end{aligned}
$$

and hence

$$
\begin{aligned}
& a \lambda\left(\pi\left(x, \theta_{1}\right) e^{a c_{x}}-\pi\left(y, \theta_{2}\right) e^{a c_{y}}\right) \frac{\left(1-\pi\left(y, \theta_{2}\right)\right) e^{-a\left(c_{x}+\bar{u}\right)}-\left(1-\pi\left(x, \theta_{1}\right)\right) e^{-a\left(c_{y}+\bar{u}\right)}}{\pi\left(x, \theta_{1}\right)-\pi\left(y, \theta_{2}\right)} \\
= & p \pi\left(x, \theta_{1}\right)-p \pi\left(x, \theta_{1}\right) e^{a c_{x}} \frac{\left(1-\pi\left(y, \theta_{2}\right)\right) e^{-a c_{x}}-\left(1-\pi\left(x, \theta_{1}\right)\right) e^{-a c_{y}}}{\pi\left(x, \theta_{1}\right)-\pi\left(y, \theta_{2}\right)}= \\
= & p \pi\left(x, \theta_{1}\right)\left[\frac{\left(1-\pi\left(x, \theta_{1}\right)\right) e^{a \Delta c}-\left(1-\pi\left(y, \theta_{2}\right)\right)+\pi\left(x, \theta_{1}\right)-\pi\left(y, \theta_{2}\right)}{\pi\left(x, \theta_{1}\right)-\pi\left(y, \theta_{2}\right)}\right]= \\
= & p \pi\left(x, \theta_{1}\right)\left[\frac{\left(1-\pi\left(x, \theta_{1}\right)\right)\left(e^{a \Delta c}-1\right)}{\pi\left(x, \theta_{1}\right)-\pi\left(y, \theta_{2}\right)}\right]>0
\end{aligned}
$$


Since the coefficient of $\lambda$ in the first term is positive, it follows that the solution for $\lambda$ of such equation is $>0$, a contradiction. Hence, it cannot be that $\bar{z}_{2}=\underline{z}_{2}$.

This completes the proof of Proposition 1.

\section{Proof of Proposition 2.}

The first best optimal contract is obtained as solution of the problem of maximizing the principal's expected profits subject to the agent's participation constraint, which under risk neutrality takes the following form:

$$
\begin{array}{rr}
\max _{\bar{w}_{1}, \underline{w}_{1}, \bar{w}_{2}, \underline{w}_{2}} \begin{array}{r}
p[ \\
\end{array} \quad \begin{array}{r}
\left.\left(x, \theta_{1}\right)\left(\bar{R}-\bar{w}_{1}\right)+\left(1-\pi\left(x, \theta_{1}\right)\right)\left(\underline{R}-\underline{w}_{1}\right)\right] \\
+(1-p)\left[\pi\left(y, \theta_{2}\right)\left(\bar{R}-\bar{w}_{2}\right)+\left(1-\pi\left(y, \theta_{2}\right)\right)\left(\underline{R}-\underline{w}_{2}\right)\right]
\end{array} \\
\text { s.t. } \begin{array}{r}
p\left[\pi\left(x, \theta_{1}\right) \bar{w}_{1}+\left(1-\pi\left(x, \theta_{1}\right)\right) \underline{w}_{1}-c_{x}\right]+ \\
(1-p)\left[\pi\left(y, \theta_{2}\right) \bar{w}_{2}+\left(1-\pi\left(y, \theta_{2}\right)\right) \underline{w}_{2}-c_{y}\right] \geq \bar{u}
\end{array}
\end{array}
$$

The maximal level of the principal's expected profits that can be attained at a solution of this problem is then clearly the one stated in the proposition and it is immediate to verify that the compensation profile given in (1) yields such level of expected profits and is then a first best optimum. It remains thus to verify the values in (1) satisfy all the incentive compatibility constraints, which under risk neutrality take the following form:

$$
\left\{\begin{array}{l}
\pi\left(x, \theta_{1}\right) \bar{w}_{1}+\left(1-\pi\left(x, \theta_{1}\right)\right) \underline{w}_{1} \geq \pi\left(x, \theta_{1}\right) \bar{w}_{2}+\left(1-\pi\left(x, \theta_{1}\right)\right) \underline{w}_{2} \\
\pi\left(x, \theta_{1}\right) \bar{w}_{1}+\left(1-\pi\left(x, \theta_{1}\right)\right) \underline{w}_{1}-c_{x} \geq \pi\left(y, \theta_{1}\right) \bar{w}_{1}+\left(1-\pi\left(y, \theta_{1}\right)\right) \underline{w}_{1}-c_{y} \\
\pi\left(x, \theta_{1}\right) \bar{w}_{1}+\left(1-\pi\left(x, \theta_{1}\right)\right) \underline{w}_{1}-c_{x} \geq \pi\left(y, \theta_{1}\right) \bar{w}_{2}+\left(1-\pi\left(y, \theta_{1}\right)\right) \underline{w}_{2}-c_{y} \\
\pi\left(y, \theta_{2}\right) \bar{w}_{2}+\left(1-\pi\left(y, \theta_{2}\right)\right) \underline{w}_{2} \geq \pi\left(y, \theta_{2}\right) \bar{w}_{1}+\left(1-\pi\left(y, \theta_{2}\right)\right) \underline{w}_{1} \\
\pi\left(y, \theta_{2}\right) \bar{w}_{2}+\left(1-\pi\left(y, \theta_{2}\right)\right) \underline{w}_{2}-c_{y} \geq \pi\left(x, \theta_{2}\right) \bar{w}_{2}+\left(1-\pi\left(x, \theta_{2}\right)\right) \underline{w}_{2}-c_{x} \\
\pi\left(y, \theta_{2}\right) \bar{w}_{2}+\left(1-\pi\left(y, \theta_{2}\right)\right) \underline{w}_{2}-c_{y} \geq \pi\left(x, \theta_{2}\right) \bar{w}_{1}+\left(1-\pi\left(x, \theta_{2}\right)\right) \underline{w}_{1}-c_{x}
\end{array}\right.
$$

This is immediate by direct substitution.

\section{Proof of Proposition 3.}

We first consider a local deviation from the contract specified in (3) such that $d \underline{w}_{1}>0$ and such that $\left(I C 3^{*}\right),\left(I C 4^{*}\right)$ and $\left(P C^{*}\right)$ continue to hold as equalities. We conjecture, and verify below, that the sign of the changes in the other wage variables is as follows, $d \bar{w}_{1}<0, d \bar{w}_{2}>0$, and $d \underline{w}_{2}<0$, and in the agent's expected utility in the two $\theta$ states is $d u\left(\theta_{1}\right)<0, d u\left(\theta_{2}\right)>0$. That is, the agent is no longer fully insured in state $\theta_{2}$ nor across states $\theta_{1}$ and $\theta_{2}$, which fixes his 'beliefs' in the incentive and participation constraints. 
Differentiating $\left(I C 3^{*}\right),\left(I C 4^{*}\right)$ and $\left(P C^{*}\right)$, written as equalities, with respect to $\bar{w}_{1}, \underline{w}_{1}, \bar{w}_{2}, \underline{w}_{2}$, and solving these equations for $d \bar{w}_{1}, d \bar{w}_{2}, d \underline{w}_{2}$, as a function of $d \underline{w}_{1}>0$ yields:

$$
\begin{aligned}
d \bar{w}_{1}= & {\left[\frac{-1}{(\hat{p}+\alpha(p))\left(\hat{\pi}\left(x, \theta_{1}\right)-\alpha\left(x, \theta_{1}\right)\right)+(1-\hat{p}-\alpha(p))\left(\hat{\pi}\left(y, \theta_{2}\right)-\alpha\left(y, \theta_{2}\right)\right)}+1\right] d \underline{w}_{1} } \\
d \bar{w}_{2}= & {\left[1-\frac{\hat{\pi}\left(x, \theta_{1}\right)-\alpha\left(x, \theta_{1}\right)-\hat{\pi}\left(y, \theta_{2}\right)+\alpha\left(y, \theta_{2}\right)+\left[\hat{\pi}\left(y, \theta_{1}\right)-\alpha\left(y, \theta_{1}\right)-\hat{\pi}\left(x, \theta_{1}\right)+\alpha\left(x, \theta_{1}\right)\right]\left[\hat{\pi}\left(y, \theta_{2}\right)-\alpha\left(y, \theta_{2}\right)\right]}{\left[(\hat{p}+\alpha(p))\left(\hat{\pi}\left(x, \theta_{1}\right)-\alpha\left(x, \theta_{1}\right)\right)+(1-\hat{p}-\alpha(p))\left(\hat{\pi}\left(y, \theta_{2}\right)-\alpha\left(y, \theta_{2}\right)\right)\right]\left[\hat{\pi}\left(y, \theta_{1}\right)-\alpha\left(y, \theta_{1}\right)-\hat{\pi}\left(y, \theta_{2}\right)+\alpha\left(y, \theta_{2}\right)\right]}\right] d \underline{w}_{1} } \\
d \underline{w}_{2}= & {\left[1-\frac{\left[\hat{\pi}\left(y, \theta_{1}\right)-\alpha\left(y, \theta_{1}\right)-\hat{\pi}\left(x, \theta_{1}\right)+\alpha\left(x, \theta_{1}\right)\right]\left[\hat{\pi}\left(y, \theta_{2}\right)-\alpha\left(y, \theta_{2}\right)\right]}{\left[(\hat{p}+\alpha(p))\left(\hat{\pi}\left(x, \theta_{1}\right)-\alpha\left(x, \theta_{1}\right)\right)+(1-\hat{p}-\alpha(p))\left(\hat{\pi}\left(y, \theta_{2}\right)-\alpha\left(y, \theta_{2}\right)\right)\right]\left[\hat{\pi}\left(y, \theta_{1}\right)-\alpha\left(y, \theta_{1}\right)-\hat{\pi}\left(y, \theta_{2}\right)+\alpha\left(y, \theta_{2}\right)\right]}\right] d \underline{w}_{1} }
\end{aligned}
$$

It is immediate to verify from the above expressions that the sign of the changes is the one conjectured.

The change in the principal's profit is given by

$$
-\left\{\hat{p}\left[\hat{\pi}\left(x, \theta_{1}\right) d \bar{w}_{1}+\left(1-\hat{\pi}\left(x, \theta_{1}\right)\right) d \underline{w}_{1}\right]+(1-\hat{p})\left[\hat{\pi}\left(y, \theta_{2}\right) d \bar{w}_{2}+\left(1-\hat{\pi}\left(y, \theta_{2}\right)\right) d \underline{w}_{2}\right]\right\}
$$

Substituting for $d \bar{w}_{1}, d \bar{w}_{2}, d \underline{w}_{2}$ the expressions found in (13) yields:

$$
\begin{aligned}
& \left\{-1+\frac{\hat{p} \hat{\pi}\left(x, \theta_{1}\right)+(1-\hat{p}) \hat{\pi}\left(y, \theta_{2}\right)}{\left[(\hat{p}+\alpha(p))\left(\hat{\pi}\left(x, \theta_{1}\right)-\alpha\left(x, \theta_{1}\right)\right)+(1-\hat{p}-\alpha(p))\left(\hat{\pi}\left(y, \theta_{2}\right)-\alpha\left(y, \theta_{2}\right)\right)\right]}\right. \\
& \left.+(1-\hat{p}) \frac{\left[\hat{\pi}\left(x, \theta_{1}\right)-\alpha\left(x, \theta_{1}\right)-\hat{\pi}\left(y, \theta_{1}\right)+\alpha\left(y, \theta_{1}\right)\right] \alpha\left(y, \theta_{2}\right)}{\left[(\hat{p}+\alpha(p))\left(\hat{\pi}\left(x, \theta_{1}\right)-\alpha\left(x, \theta_{1}\right)\right)+(1-\hat{p}-\alpha(p))\left(\hat{\pi}\left(y, \theta_{2}\right)-\alpha\left(y, \theta_{2}\right)\right)\right]\left[\hat{\pi}\left(y, \theta_{1}\right)-\alpha\left(y, \theta_{1}\right)-\hat{\pi}\left(y, \theta_{2}\right)+\alpha\left(y, \theta_{2}\right)\right]}\right\} d \underline{w}_{1}
\end{aligned}
$$

Since $d \underline{w}_{1}>0$ the above term is negative, that is the deviation considered is not profitable if and only if the term appearing in curly brackets is positive, that is (4) holds.

The other possible deviation, with $d \underline{w}_{1}<0$, can be treated in a similar fashion. The wage changes have here the opposite sign as above, hence the induced beliefs need to be modified accordingly. The expression for the change in expected profits in that case is then:

$$
\begin{aligned}
& \left\{-1-\frac{\hat{p} \hat{\pi}\left(x, \theta_{1}\right)+(1-\hat{p}) \hat{\pi}\left(y, \theta_{2}\right)}{\left[(\hat{p}-\alpha(p))\left(\hat{\pi}\left(x, \theta_{1}\right)-\alpha\left(x, \theta_{1}\right)\right)+(1-\hat{p}+\alpha(p))\left(\hat{\pi}\left(y, \theta_{2}\right)-\alpha\left(y, \theta_{2}\right)\right)\right]}\right. \\
& -(1-\hat{p}) \frac{\hat{\pi}\left(y, \theta_{2}\right)\left[\hat{\pi}\left(y, \theta_{1}\right)+\alpha\left(y, \theta_{1}\right)-2 \alpha\left(y, \theta_{2}\right)-\hat{\pi}\left(x, \theta_{1}\right)-\alpha\left(x, \theta_{1}\right)\right]}{\left[(\hat{p}-\alpha(p))\left(\hat{\pi}\left(x, \theta_{1}\right)-\alpha\left(x, \theta_{1}\right)\right)+(1-\hat{p}+\alpha(p))\left(\hat{\pi}\left(y, \theta_{2}\right)-\alpha\left(y, \theta_{2}\right)\right)\right]\left[\hat{\pi}\left(y, \theta_{1}\right)+\alpha\left(y, \theta_{1}\right)-\hat{\pi}\left(y, \theta_{2}\right)-\alpha\left(y, \theta_{2}\right)\right]} \\
& \left.-(1-\hat{p}) \frac{-\left(\hat{\pi}\left(y, \theta_{2}\right)-\alpha\left(y, \theta_{2}\right)\right)\left(\hat{\pi}\left(y, \theta_{1}\right)+\alpha\left(y, \theta_{1}\right)\right)+\left(\hat{\pi}\left(y, \theta_{2}\right)+\alpha\left(y, \theta_{2}\right)\right)\left(\hat{\pi}\left(x, \theta_{1}\right)-\alpha\left(x, \theta_{1}\right)\right)}{\left[(\hat{p}-\alpha(p))\left(\hat{\pi}\left(x, \theta_{1}\right)-\alpha\left(x, \theta_{1}\right)\right)+(1-\hat{p}+\alpha(p))\left(\hat{\pi}\left(y, \theta_{2}\right)-\alpha\left(y, \theta_{2}\right)\right)\right]\left[\hat{\pi}\left(y, \theta_{1}\right)+\alpha\left(y, \theta_{1}\right)-\hat{\pi}\left(y, \theta_{2}\right)-\alpha\left(y, \theta_{2}\right)\right]}\right\} d \underline{w}_{1}
\end{aligned}
$$

The principal would benefit from this deviation only if the term appearing in curly brackets is negative (as in this case $d \underline{w}_{1}<0$ ). This term is negative if and only if

$$
\begin{aligned}
& \left(\hat{\pi}\left(y, \theta_{2}\right)+\alpha\left(y, \theta_{2}\right)-\hat{\pi}\left(y, \theta_{1}\right)-\alpha\left(y, \theta_{1}\right)\right) \times \\
& {\left[(\hat{p}-\alpha(p))\left(\hat{\pi}\left(x, \theta_{1}\right)-\alpha\left(x, \theta_{1}\right)\right)+(1-\hat{p}-\alpha(p))\left(\hat{\pi}\left(y, \theta_{2}\right)-\alpha\left(y, \theta_{2}\right)\right)\right]-} \\
& (1-\hat{p}) \alpha\left(y, \theta_{2}\right)\left[\hat{\pi}\left(x, \theta_{1}\right)-\alpha\left(x, \theta_{1}\right)-\hat{\pi}\left(y, \theta_{1}\right)-\alpha\left(y, \theta_{1}\right)\right]>0
\end{aligned}
$$


It can be shown that the expression on the left hand side of the above inequality is bounded above by

$-\alpha\left(x, \theta_{1}\right) \hat{p}\left(\hat{\pi}\left(y, \theta_{2}\right)+\alpha\left(y, \theta_{2}\right)-\hat{\pi}\left(y, \theta_{1}\right)-\alpha\left(y, \theta_{1}\right)\right)-(1-\hat{p}) \alpha\left(y, \theta_{2}\right)\left[\hat{\pi}\left(x, \theta_{1}\right)-\alpha\left(x, \theta_{1}\right)-\hat{\pi}\left(y, \theta_{2}\right)-\alpha\left(y, \theta_{2}\right)\right]$

which is always negative. Hence, the considered deviation is never optimal.

We now prove that at an optimal flexible contract we have $\bar{w}_{2}>\underline{w}_{2}$ if condition (4) does not hold. Note first that $\bar{w}_{2}<\underline{w}_{2}$ cannot be part of a solution, since it would then pay for the principal to reduce the volatility in $w_{2}$. Assume hence that (4) does not hold and that $\bar{w}_{2}=\underline{w}_{2}$.

Given that $w_{2}$ is constant, $\left(I C_{1}^{*}\right)$ is implied by $\left(I C_{3}^{*}\right)$ and $\left(I C_{5}^{*}\right)$ always holds. Observe next that $\left(I C_{3}^{\star}\right)$ holds with equality since otherwise one could increase the principal's profit by lowering wages in state $\theta_{1}$ while increasing $w_{2}$ in a way that preserves $\left(I C_{2}^{*}\right)$ and $(P C)$ (as well as $\left(I C_{4}^{*}\right)$ and $\left(I C_{6}^{*}\right)$ ). This implies that the agent's expected utility is the same in state $\theta_{1}$ and in state $\theta_{2}$.

Furthermore, $\left(I C_{3}^{*}\right)$ binding implies that $\left(I C_{6}^{*}\right)$ is slack and, together with $\left(I C_{4}^{*}\right)$, that $\left(I C_{2}^{*}\right)$ holds. Now, if $\left(I C_{4}^{*}\right)$ were slack, it would be possible to lower $\bar{w}_{1}$ and increase $\underline{w}_{1}$ so that the expected utility of the agent in state 1 is constant while the profit for the principal increases.

Hence, if $w_{2}$ constant is to be part of the solution, $\left(I C_{4}^{*}\right)$ has to bind. But then, the optimal contract is the one specified in (3) (that satisfies $\left(I C_{3}^{*}\right),\left(I C_{4}^{*}\right)$ and $\left(\mathrm{PC}^{*}\right)$ as equality with $w_{2}$ constant), and we just proved that it is not optimal when condition (4) does not hold. Hence the contradiction: when (4) does not hold, it has to be the case that $w_{2}$ is non constant.

\section{Proof of Corollary 2.}

For the open set of parameter values for which the contract described in (3) is the optimal flexible contract, the expected wage bill the principal has to pay is

$$
\hat{p}\left(\hat{u}+c_{x}\right)+(1-\hat{p})\left(\bar{u}+c_{y}\right)+\hat{p} \frac{\alpha\left(x, \theta_{1}\right)}{\hat{\pi}\left(x, \theta_{1}\right)-\hat{\pi}\left(y, \theta_{2}\right)-\left(\alpha\left(x, \theta_{1}\right)-\alpha\left(y, \theta_{2}\right)\right)} \Delta c
$$

Recall that $\alpha\left(x, \theta_{1}\right)=\alpha \frac{\bar{\pi}\left(x, \theta_{1}\right)-\underline{\pi}\left(x, \theta_{1}\right)}{2}$ and $\alpha\left(y, \theta_{2}\right)=\alpha \frac{\bar{\pi}\left(y, \theta_{2}\right)-\underline{\pi}\left(y, \theta_{2}\right)}{2}$ and substitute these terms in the above expression. If we then differentiate the above expression with respect 
to $\alpha$ we readily see that the expected wage bill is always increasing in $\alpha$. Increasing the degree of imprecision aversion will therefore lower expected profits at the flexible contract. The same is clearly true for increases in the imprecision of the return to action $x$ in state $\theta_{1}$, that is of the width of the interval $\left[\bar{\pi}\left(x, \theta_{1}\right)-\underline{\pi}\left(x, \theta_{1}\right)\right]$

\section{Further results for the numerical example of Section 3.3}

\section{The effect of risk aversion on wage and utility differentials}

We illustrate the implications that the level of the agent's degree of risk aversion has for the specific properties of the optimal flexible contract. Figure 2 describes the effect of varying $a$ on the spread between the compensation paid for the high and low output realizations at the optimal flexible contract respectively in state $\theta_{1}$ (i.e. $\bar{w}_{1}-\underline{w}_{1}$ ) and $\theta_{2}$. Figure 3 shows then the effect on the utility differential. We see that both the spread in state $\theta_{1}$ and the utility differential vary non monotonically with $a$, first increasing and then decreasing.

\section{INCLUDE FIGURE 2 AND 3 ABOUT HERE}

We should point out however that these properties - unlike the effect on the relative profitability of flexible and rigid contracts displayed in Figure 1 - are not quite robust to changes in the values of the parameters considered in Table 1. For different parameter values, other patterns of the utility and the wage differential, for instance monotonically decreasing in state $\theta_{1}$, may obtain.

\section{The effect of actions' productivity and cost}

We investigate next how the relative profitability of flexible versus rigid contracts is affected by the following parameters: the levels of the probability of success for each action and event in which it is undertaken and the cost of the different types of actions $c_{z}$. Our findings, still based on the parametrization described in Table 1, are summarized in Table 2. A + (resp. -) sign indicates that an increase in the parameter value indicated in the top of the column always increases (decreases) the variable appearing in the row, while a ? indicates the effect is ambiguous, not always of the same sign.

For instance, the first column reports the sign of the effects of increasing $\pi\left(x, \theta_{1}\right)$, within the interval indicated, $[.75, .9]$ on the following variables: (i) the differential between the expected profits at the optimal flexible contract and those at the $x$ rigid contract in 


\begin{tabular}{c|cccc|cc}
$\begin{array}{c}\text { Parameter } \\
\text { Range }\end{array}$ & $\pi\left(x, \theta_{1}\right)$ & $\pi\left(x, \theta_{2}\right)$ & $\pi\left(y, \theta_{1}\right)$ & $\pi\left(y, \theta_{2}\right)$ & $c_{x}$ & $c_{y}$ \\
{$[.75, .9]$} & {$[.35, .55]$} & {$[.15, .25]$} & {$[.3, .5]$} & {$[1.2,1.8]$} & {$[.7,1.25]$} \\
\hline \hline Profit flexible - profit $x$ & + & - & - & + & $?$ & $?$ \\
\hline Profit flexible - profit $y$ & + & $=$ & - & - & - & + \\
\hline $\bar{w}_{1}-\underline{w}_{1}$ & - & $=$ & + & $?$ & + & - \\
\hline $\bar{w}_{2}-\underline{w}_{2}$ & - & $=$ & - & + & + & - \\
\hline$u\left(\theta_{2}\right)-u\left(\theta_{1}\right)$ & - & $=$ & - & + & + & -
\end{tabular}

Table 2: Comparative statics with respect to probabilities and costs

the first row and at the $y$ rigid contract in the second row; (ii) the spread between the compensation paid for the high and low realization of the output when state $\theta_{1}$ occurs in the third row and when $\theta_{2}$ occurs in the fourth one; (iii) the difference in expected utility in the two states. All this when the other parameters are kept fixed at the values indicated in Table 1.

In particular, we find that the profitability of the flexible contract, relative to both rigid contracts, increases if $\pi\left(x, \theta_{1}\right)$ (probability of success with action $x$ in state 1 ) increases, or $\pi\left(y, \theta_{1}\right)$ decreases. Such changes increase the productivity of the costlier action $(x)$ relative to the less costly one in state $\theta_{1}$ as well as the variance of the productivity of each action across the different states. The same effects are obtained with a decrease in $\pi\left(x, \theta_{2}\right)$, reducing the difference between the productivity of actions $x$ and $y$ in state $\theta_{2}$.

We also see that the variability in the compensation paid in state $\theta_{2}$, where the less costly action is implemented, always moves in the same direction as the utility differential $u\left(\theta_{2}\right)-u\left(\theta_{1}\right)$, suggesting these two are complementary instruments to address the incentive problems generated by the private information over $\theta$, as already mentioned in Remark 2 . 


\section{Online Appendix - NOT TO BE INCLUDED IN PRINTED VERSION}

Proposition B.1: $\quad$ When $\theta$ is commonly observable, under Assumption 2 the optimal contract still implements actions $x$ and $y$ and is characterized as follows:

$$
\begin{aligned}
e^{-a \bar{w}_{1}} & =\frac{\left(1-\pi\left(y, \theta_{1}\right)\right) e^{-a\left(\bar{u}+c_{x}\right)}-\left(1-\pi\left(x, \theta_{1}\right)\right) e^{-a\left(\bar{u}+c_{y}\right)}}{\pi\left(x, \theta_{1}\right)-\pi\left(y, \theta_{1}\right)} \\
e^{-a \underline{w}_{1}} & =\frac{\pi\left(x, \theta_{1}\right) e^{-a\left(\bar{u}+c_{y}\right)}-\pi\left(y, \theta_{1}\right) e^{-a\left(\bar{u}+c_{x}\right)}}{\pi\left(x, \theta_{1}\right)-\pi\left(y, \theta_{1}\right)} \\
\bar{w}_{2} & =\underline{w}_{2}=\bar{u}+c_{y}
\end{aligned}
$$

Thus the agent's expected utility is the same in $\theta_{1}$ and $\theta_{2}$.

Proof of Proposition B.1. Observe first that (IC2) implies that $\bar{w}_{1}>\underline{w}_{1}$. The property $\bar{w}_{2}=\underline{w}_{2}$ can then be easily verified and ensures that (IC5) is always satisfied. Consider then the first order conditions of problem of maximizing the principal's expected revenue subject to $(\mathrm{IC} 2)$, and $(\mathrm{PC}):^{25}$

$$
\left\{\begin{array}{c}
(i) \quad-p \pi\left(x, \theta_{1}\right)+\lambda_{I C}\left(-a \pi\left(y, \theta_{1}\right) e^{-a\left(\bar{w}_{1}-c_{y}\right)}+a \pi\left(x, \theta_{1}\right) e^{-a\left(\bar{w}_{1}-c_{x}\right)}\right)+ \\
\lambda_{P C} a p \pi\left(x, \theta_{1}\right) e^{-a\left(\bar{w}_{1}-c_{x}\right)}=0 \\
(i i) \quad-p\left(1-\pi\left(x, \theta_{1}\right)\right)+\lambda_{I C}\left(-a\left(1-\pi\left(y, \theta_{1}\right)\right) e^{-a\left(\underline{w}_{1}-c_{y}\right)}+a\left(1-\pi\left(x, \theta_{1}\right)\right) e^{-a\left(\underline{w}_{1}-c_{x}\right)}+\right. \\
\lambda_{P C} a p\left(1-\pi\left(x, \theta_{1}\right)\right) e^{-a\left(\underline{w}_{1}-c_{x}\right)}=0 \\
(i i i) \quad-(1-p)+(1-p) \lambda_{P C} a e^{-a\left(w_{2}-c_{y}\right)}=0
\end{array}\right.
$$

where $\lambda_{I C}, \lambda_{P C}$ are the Lagrange multipliers attached to constraints (IC2), (PC).

Condition (iii) implies that $\lambda_{P C}=\frac{e^{a\left(w_{2}-c_{y}\right)}}{a}>0$ and hence that (PC) is binding. Take now the summation of (i) and (ii) and use the complementary slackness condition (requiring that $\lambda_{I C} \times(I C)=0$ ), to obtain:

$$
-p+a \lambda_{P C} p\left[\pi\left(x, \theta_{1}\right) e^{-a\left(\bar{w}_{1}-c_{x}\right)}+\left(1-\pi\left(x, \theta_{1}\right)\right) e^{-a\left(\underline{w}_{1}-c_{x}\right)}\right]=0
$$

Using the fact that $(\mathrm{PC})$ is binding, this amounts to:

$$
-p+a \lambda_{P C}\left[e^{-a \bar{u}}-(1-p) e^{-a\left(w_{2}-c_{y}\right)}\right]=0
$$

\footnotetext{
${ }^{25}$ Rewrite (IC2) as follows

$$
e^{-a \bar{w}_{1}}\left[\pi\left(x, \theta_{1}\right) e^{a c_{x}}-\pi\left(y, \theta_{1}\right) e^{a c_{y}}\right] \leq e^{-a \underline{w}_{1}}\left[\left(1-\pi\left(y, \theta_{1}\right)\right) e^{a c_{y}}-\left(1-\pi\left(x, \theta_{1}\right)\right) e^{a c_{x}}\right]
$$
}

A necessary condition for this to hold and hence for the existence of a solution to the principal's programme is $e^{a \Delta c} \leq \frac{1-\pi\left(y, \theta_{1}\right)}{1-\pi\left(x, \theta_{1}\right)}$. 
and finally, $e^{-a\left(w_{2}-c_{y}\right)}=e^{-a \bar{u}}$. Using again the fact that $(\mathrm{PC})$ binds, we obtain that

$$
\pi\left(x, \theta_{1}\right) e^{-a\left(\bar{w}_{1}-c_{x}\right)}+\left(1-\pi\left(x, \theta_{1}\right)\right) e^{-a\left(\underline{w}_{1}-c_{x}\right)}=e^{-a \bar{u}}=e^{-a\left(w_{2}-c_{y}\right)},
$$

thus establishing the fact that at a solution of the above problem the utility of the agent is the same in state $\theta_{1}$ and $\theta_{2}$, in contrast with the property established in Proposition 1 for the optimal flexible contract when $\theta$ is only privately observed by the agent.

Proposition B.2: Assume the condition in Assumption 2.ii) is replaced by the following: $\pi\left(x, \theta_{1}\right)>\pi\left(x, \theta_{2}\right)>\pi\left(y, \theta_{1}\right)>\pi\left(y, \theta_{2}\right)$ (or by $\pi\left(x, \theta_{1}\right)>\pi\left(y, \theta_{1}\right)>\pi\left(x, \theta_{2}\right)>$ $\left.\pi\left(y, \theta_{2}\right)\right)$ Then the optimal flexible contract is the same as the optimal contract obtained when $\theta$ is observable. On the other hand, this not true under the condition in Assumption 2.ii), or when $\pi\left(x, \theta_{2}\right)>\pi\left(x, \theta_{1}\right)>\pi\left(y, \theta_{2}\right)>\pi\left(y, \theta_{1}\right)$, or $\pi\left(x, \theta_{2}\right)>$ $\pi\left(y, \theta_{2}\right)>\pi\left(x, \theta_{1}\right)>\pi\left(y, \theta_{1}\right)$.

\section{Proof of Proposition B.2}

To establish the result it suffices to show that the optimal flexible contract obtained in Proposition B.1 remains feasible when $\theta$ is unobservable and $\pi\left(x, \theta_{1}\right)>\pi\left(x, \theta_{2}\right)>$ $\pi\left(y, \theta_{1}\right)>\pi\left(y, \theta_{2}\right)$ holds, that is, it satisfies all the remaining constraints, (IC1, IC3, IC4, IC6).

It follows from the proof of Proposition B.1 that the optimal contract when $\theta$ is observable satisfies (IC2) and the two following conditions (corresponding to (PC) when $\bar{w}_{2}=\underline{w}_{2}$ and $\left.u\left(\theta_{1}\right)=u\left(\theta_{2}\right)\right)$ :

(a) $\pi\left(x, \theta_{1}\right) e^{-a\left(\bar{w}_{1}-c_{x}\right)}+\left(1-\pi\left(x, \theta_{1}\right)\right) e^{-a\left(\underline{w}_{1}-c_{x}\right)}=e^{-a \bar{u}}$

(b) $e^{-a\left(w_{2}-c_{y}\right)}=e^{-a \bar{u}}$

(IC1), given (a) and (b), amounts to $e^{-a \bar{u}} \leq e^{-a \bar{u}} e^{a \Delta c}$, which is satisfied given that $\Delta c>0$.

(IC3), given (a) and (b), amounts to $e^{-a \bar{u}} \leq e^{-a \bar{u}}$, and hence also holds.

(IC4), given (b), amounts to $-e^{-a \bar{u}} \geq-\pi\left(y, \theta_{2}\right) e^{-a\left(\bar{w}_{1}-c_{y}\right)}-\left(1-\pi\left(y, \theta_{2}\right)\right) e^{-a\left(\underline{w}_{1}-c_{y}\right)}$. Using condition (a), this inequality can be equivalently written as $-\pi\left(x, \theta_{1}\right) e^{-a\left(\bar{w}_{1}-c_{x}\right)}-(1-$ $\left.\pi\left(x, \theta_{1}\right)\right) e^{-a\left(\underline{w}_{1}-c_{x}\right)} \geq-\pi\left(y, \theta_{2}\right) e^{-a\left(\bar{w}_{1}-c_{y}\right)}-\left(1-\pi\left(y, \theta_{2}\right)\right) e^{-a\left(\underline{w}_{1}-c_{y}\right)}$. Since $\pi\left(y, \theta_{1}\right)>\pi\left(y, \theta_{2}\right)$ and $\bar{w}_{1}>\underline{w}_{1}$, we have $-\pi\left(y, \theta_{1}\right) e^{-a\left(\bar{w}_{1}-c_{y}\right)}-\left(1-\pi\left(y, \theta_{1}\right)\right) e^{-a\left(\underline{w}_{1}-c_{y}\right)}>-\pi\left(y, \theta_{2}\right) e^{-a\left(\bar{w}_{1}-c_{y}\right)}-$ 
$\left(1-\pi\left(y, \theta_{2}\right)\right) e^{-a\left(\underline{w}_{1}-c_{y}\right)}$, and hence, using $(\mathrm{IC} 2)$, we get $-\pi\left(x, \theta_{1}\right) e^{-a\left(\bar{w}_{1}-c_{x}\right)}-\left(1-\pi\left(x, \theta_{1}\right)\right) e^{-a\left(\underline{w}_{1}-c_{x}\right)} \geq$ $-\pi\left(y, \theta_{2}\right) e^{-a\left(\bar{w}_{1}-c_{y}\right)}-\left(1-\pi\left(y, \theta_{2}\right)\right) e^{-a\left(\underline{w}_{1}-c_{y}\right)}$. Given the argument above, this ensures that (IC4) is satisfied. Notice that this result does not hold if, instead of the condition $\pi\left(x, \theta_{1}\right)>\pi\left(x, \theta_{2}\right)>\pi\left(y, \theta_{1}\right)>\pi\left(y, \theta_{2}\right)$, we impose Assumption 2.ii).

(IC6), given (a) and (b), amounts to $-e^{-a \bar{u}}=-\pi\left(x, \theta_{1}\right) e^{-a\left(\bar{w}_{1}-c_{x}\right)}-\left(1-\pi\left(x, \theta_{1}\right)\right) e^{-a\left(\underline{w}_{1}-c_{x}\right)}>$ $-\pi\left(x, \theta_{2}\right) e^{-a\left(\bar{w}_{1}-c_{x}\right)}-\left(1-\pi\left(x, \theta_{2}\right)\right) e^{-a\left(\underline{w}_{1}-c_{x}\right)}$, which is satisfied given that $\pi\left(x, \theta_{1}\right)>$ $\pi\left(x, \theta_{2}\right)$ and $\bar{w}_{1}>\underline{w}_{1}$.

We have thus shown that, when $\pi\left(x, \theta_{1}\right)>\pi\left(x, \theta_{2}\right)>\pi\left(y, \theta_{1}\right)>\pi\left(y, \theta_{2}\right)$, the optimal contract for the case where $\theta$ is observable is a feasible contract also when $\theta$ is unobservable. Hence it is the optimal contract also in that case.

It is immediate to verify that the same argument holds when $\pi\left(x, \theta_{1}\right)>\pi\left(y, \theta_{1}\right)>$ $\pi\left(x, \theta_{2}\right)>\pi\left(y, \theta_{2}\right)$. In contrast, both (IC4) and (IC6) are violated when $\pi\left(x, \theta_{2}\right)>$ $\pi\left(x, \theta_{1}\right)>\pi\left(y, \theta_{2}\right)>\pi\left(y, \theta_{1}\right)$, or $\pi\left(x, \theta_{2}\right)>\pi\left(y, \theta_{2}\right)>\pi\left(x, \theta_{1}\right)>\pi\left(y, \theta_{1}\right)$. 


\section{Figures}
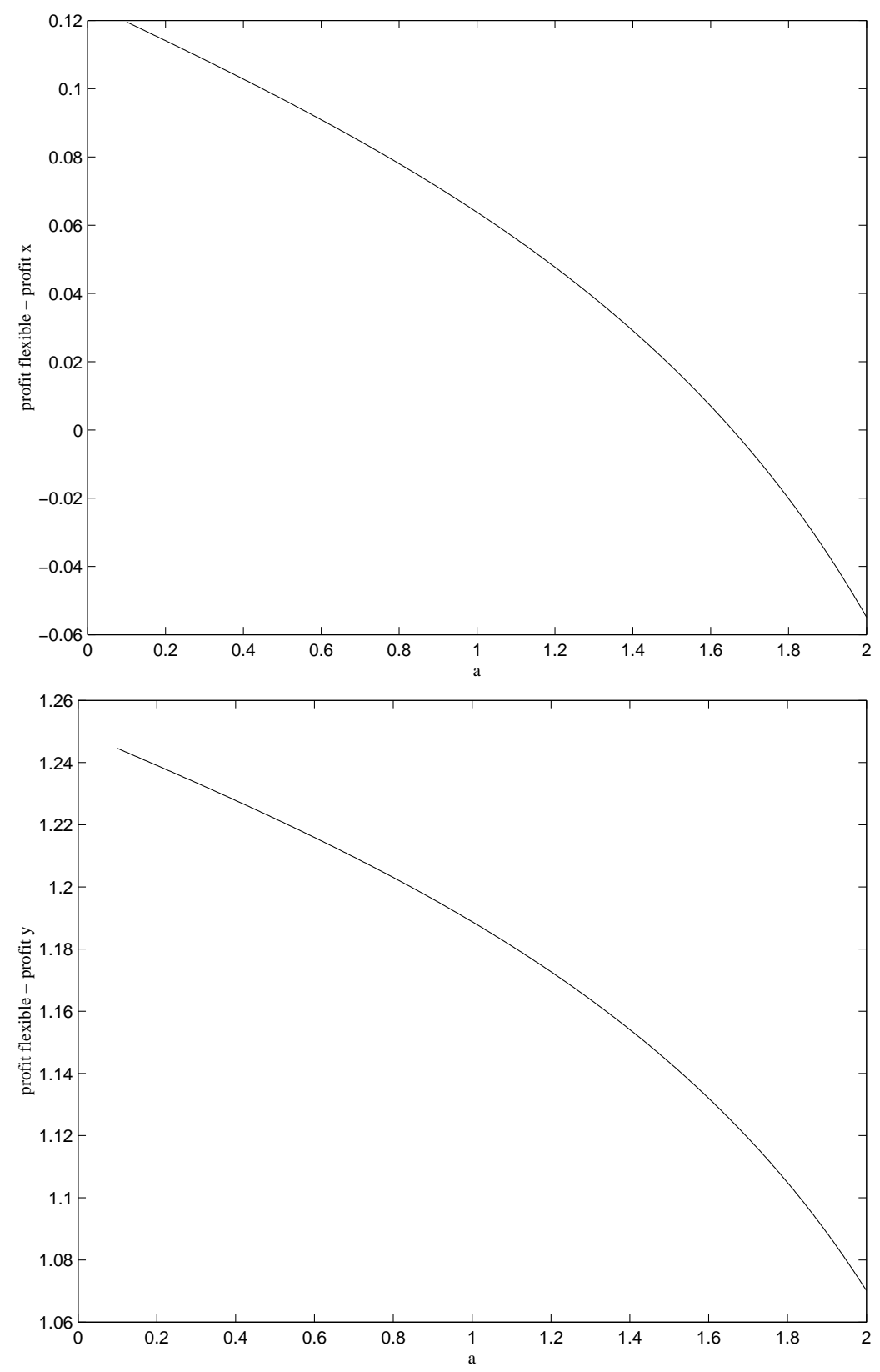

Figure 1: Profit differential between the flexible and rigid contracts as a function of risk aversion 

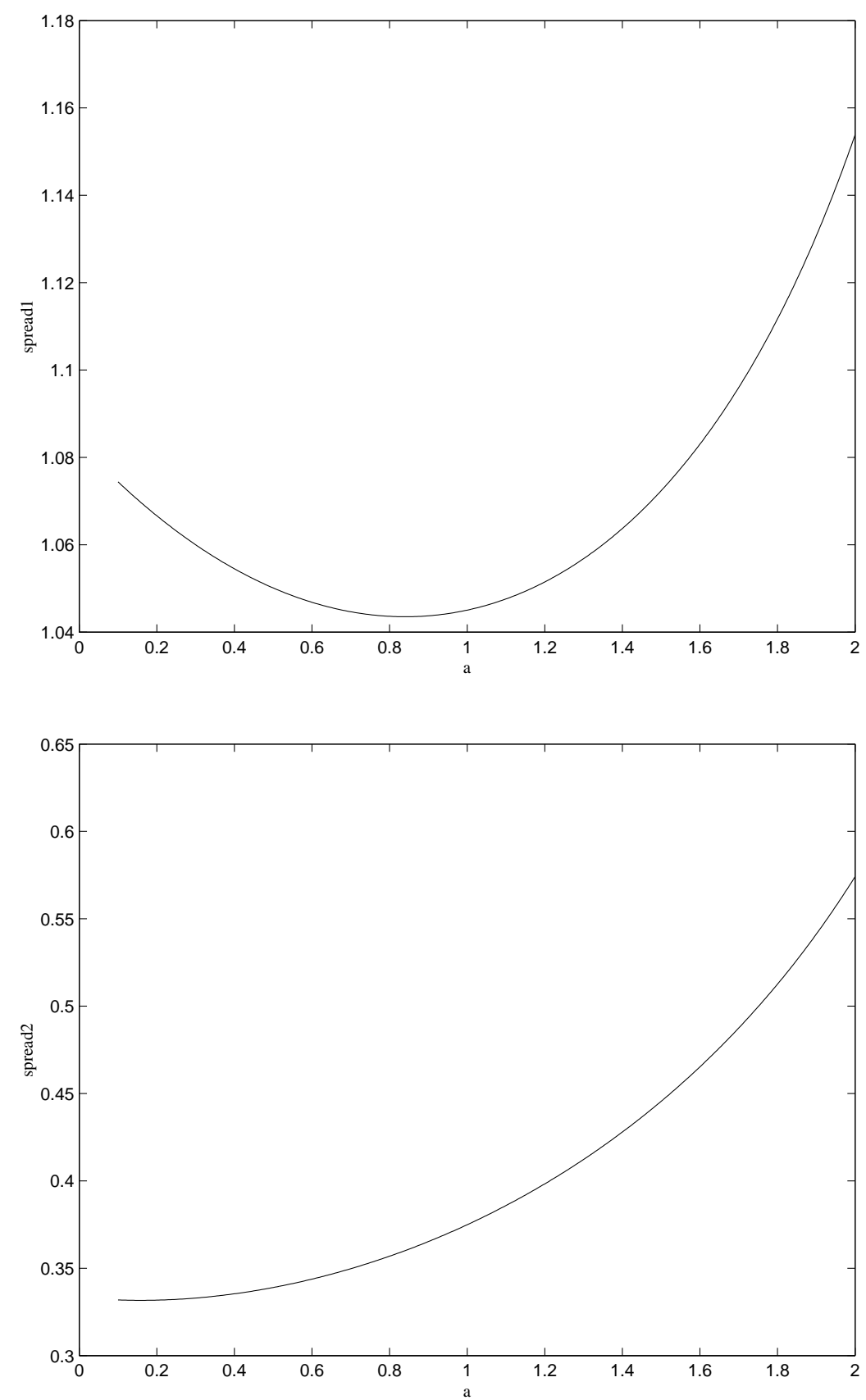

Figure 2: Wage differentials at the optimal flexible contract as a function of risk aversion 


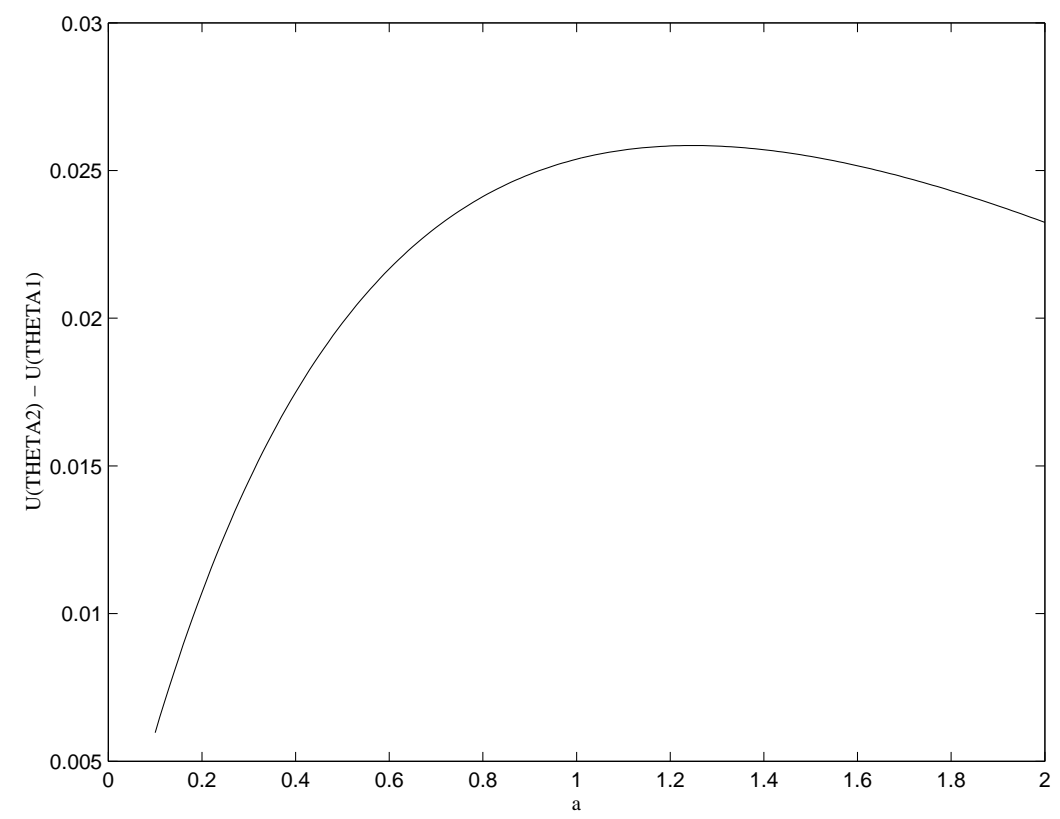

Figure 3: Utility differential $u\left(\theta_{2}\right)-u\left(\theta_{1}\right)$ at the optimal flexible contract as a function of $a$ 